\title{
Arreglo de los Polígonos del Exocorion de Huevos Eclosionados de Algunas Especies de los géneros Triatoma Laporte, Meccus Stal y Nesotriatoma Usinger (Heteroptera: Reduviidae)
}

\author{
Gleyni González¹, Elis Aldana', Eliézer Lizano \& Glauco López²
}

1. Universidad de Los Andes, Departamento de Biología Facultad de Ciencias, e-mail: labenthl@ula.ve, aldana@ula.ve (Autor para correspondência). 2. Universidad de Los Andes, Departamento de Matemáticas Facultad de Ciencias. e-mail: glauco@ula.ve

EntomoBrasilis 2 (3): 76-89 (2009)

Resumen. En este trabajo se estudiaron estructural y cuantitativamente las celdas exocoriales de huevos eclosionados de Triatoma infestans (Klug), Triatoma lenti Sherlock \& Serafim, Triatoma maculata (Erichson), Triatoma sordida (Stal), Meccus picturatus (Usinger), Meccus longipennis (Usinger), Meccus pallidipennis (Stal) y Nesotriatoma flavida (Neiva). Los huevos se analizaron por regiones: caudal, media y cefálica. Se encontró que el hexágono fue el polígono más abundante en todas las regiones del huevo y en todas las especies, seguido del pentágono y del heptágono; se encontraron diferencias estadísticamente significativas en el número tanto de hexágonos y pentágonos entre regiones de una misma especie y entre la misma región de las diferentes especies, sin embargo, el número promedio de heptágonos no varió al comparar la región media entre las diferentes especies y al comparar las diferentes regiones en una misma especie. No se encontraron diferencias estadísticamente significativas en el promedio de polígonos, segmentos totales, heptágonos, hexágonos y pentágonos, al comparar las especies examinadas del género Meccus. Se propone un protocolo de identificación y análisis de teselaciones, con el cual se encontró que en 108 campos examinados de $385.000 \mu \mathrm{m}^{2}$ cada uno, se identificaron teselaciones compartidas en los 108 campos observados con determinadas combinaciones de heptágonos, hexágonos y pentágonos, y se observaron teselaciones no compartidas en la totalidad de los campos, con combinaciones de polígonos de cuatro a nueve lados, y cada teselación se representó por un grafo. Se discuten las implicaciones taxonómicas, funcionales, filogenéticas y construccionales de los arreglos poligonales descritos.

Palabras-clave: Exocorion, Morfología, Teoría de Grafos, Teselación, Ultraestructura

\section{Arragement of exochorial polygons of hatched eggs in several species of the genera Triatoma Laporte, Meccus Stal y Nesotriatoma Usinger (Heteroptera: Reduviidae).}

Abstract. In this study a structural and quantitative analysis of the exochorial polygonal cells of hatched eggs of Triatoma infestans (Klug), Triatoma lenti Sherlock \& Serafim, Triatoma maculata (Erichson), Triatoma sordida (Stal), Meccus picturatus (Usinger), Meccus longipennis (Usinger), Meccus pallidipennis (Stal) and Nesotriatoma flavida (Neiva) was performed; cephalic, median and caudal egg regions were compared. It was found that cells with hexagonal shape were more abundant among all egg regions and studied species, followed by pentagonal and heptagonal polygons respectively. The average number of hexagonal and pentagonal polygons was significantly different among the three egg regions for each species, and when comparing the median, cephalic and caudal egg regions among different species. However, the average number of heptagons did not differ among the three egg regions for each species. The average number of heptagons did not differ when comparing the median egg region, but differed when comparing the cephalic and caudal egg regions, among different species. The average number of hexagons, pentagons, heptagons, total polygons, and total segments did not differ among the different species eggs of Meccus genus. A group of 108 micrographies $\left(380,000 \mu \mathrm{m}^{2}\right.$ each one) were studied using tessellation methods. Shared tilings and unshared tilings were found in all the micrographies observed. Shared tilings were conformed by combinations of heptagons, hexagons and pentagons, and unshared tilings by combinations of polygons from four to nine sides. Finally, each tiling arrangement was represented as a graph. Taxonomic, functional and phylogenetic implications of the polygonal cell arrangement described here are discussed.

Keywords: Exochorion, Graph Theory, Morphology, Tessellation, Ultrastructure.

$\mathbf{E}$ n el género Triatoma Laporte 1832 GALVÃO et al. (2003) han reconocido 67 especies. Los adultos de las especies de este género son de color variable, los tubérculos anteníferos están situados en el medio de la región anteocular, el primer segmento del rostro es invariablemente más corto que el segundo.

Según LENT \& WYGODZINSKY (1979), dado que el género Triatoma está definido principalmente por caracteres plesiomórficos, posiblemente constituye un grupo parafilético; el género además contenía a varios complejos específicos de los cuales los complejos phyllosoma y flavida fueron recientemente convertidos a los géneros Meccus Stal, 1859 y Nesotriatoma Usinger, 1944 (CARCAVALLO et al. 1998; GALVÃO et al. 2003). Las evidencias moleculares sugieren también que Triatoma es un grupo parafilético, al tiempo que validan al género Meccus como grupo monofilético (HYPSA et al. 2002). Recientemente la hipótesis del origen polifilético de la tribu Triatomini fue apoyada al combinar las evidencias moleculares y bioquímicas con las tradicionales morfológicas y ecológicas (TARTAROTTI et al. 2006).

En los estudios filogenéticos del género Triatoma se han empleado caracteres morfológicos y moleculares (TARTAROTTI et al. 2006; HYPSA et al. 2002; CARCAVALLO et al 1998; GARCÍA 1998), pero poco se sabe de la importancia filogenética de caracteres en un estadio como lo es el huevo. Los huevos de Triatoma son simétricos y de forma elíptica, miden en promedio 2,05 $\mathrm{mm}$ de longitud y 1,14mm de diámetro, el opérculo es casi plano, no presenta cuello, y está bien marcado por una línea 
transversal (BRICEÑO-IRAGORRY 1934; BARATA 1998). Los estudios morfométricos realizados en Triatoma circummaculata (Stal) y Triatoma rubrovaria (Blanchard) revelan que los huevos no eclosionados son de mayor longitud y anchura que los eclosionados (DA ROSA et al. 2000).

El corion está constituido por dos capas; una interna llamada endocorion y una externa llamada exocorion (BEAMENT 1946), el corion es secretado por el epitelio folicular del ovario, el cual deja impresiones características sobre el exocorion (WIGGLESWORTH 1972), las impresiones son formas geométricas observables por microscopia óptica (BRICEÑOIRAGORRY 1934).

La Microscopia Electrónica de Barrido permitió revelar la morfología característica de estas impresiones denominándoseles microestructuras del exocorion (LUCENA \& REGO 1969) sugiriéndose a ésta como un carácter especie especifico (BARTH \& MUTH 1958; LENT \& WYGODZINSKY 1979; BARATA 1981); la microestructura del exocorion permitió dividir al género Triatoma en dos grupos, uno más numeroso, constituido por especies con exocorion de células hexagonales, las cuales presentan perforaciones de diferentes tamaños y distribución, y uno menos numeroso, conformado por especies que no presentan una arquitectura definida en su exocorion (BARATA 1998).

La morfología de las celdas, específicamente el promedio y la distribución de las puntuaciones internas ha revelado una diferenciación morfo-estructural del exocorion a nivel de cuatro poblaciones de Triatoma brasiliensis Neiva (COSTA et al. 1997). La morfología del exocorion además ha discriminado entre huevos de especies morfológicamente muy similares como T. maculata (Erichson) y Triatoma pseudomaculata Correa \& Espinola (GONCALVES et al. 1985) y especies con igual distribución geográfica como Triatoma vandae Carcavallo, Jurberg, Rocha, Galvão, Noireau \& Lent y Triatoma williami Galvão, Souza \& Lima (SILVA et al. 2005).

Las observaciones de los patrones geométricos en tres regiones del huevo (cefálica, media y caudal) en distintos grados del desarrollo embrionario y en huevos eclosionados de Rhodnius prolixus Stal y Triatoma patagonica Del Ponte (CHAVES \& AÑES 2003; VISCIARELLI et al. 2004 ) indican que éstos no varían, además de sugerir cualitativamente que las regiones cefálica y caudal comparten patrones geométricos con células hexagonales, pentagonales y heptagonales mientras que en la región media, dominan las células de tipo hexagonales.

Los estudios morfológicos e histológicos del exocorion realizado en huevos de los géneros Triatoma y Meccus ratificaron los ordenamientos filogenéticos generados empleando la morfología tradicional del adulto y la comparación de secuencias de ADN (HYPSA et al. 2002; OBARA et al. $2000 \mathrm{a}, \mathrm{b}$ ).

La morfología del exocorion también se ha empleado en otros grupos de insectos con fines taxonómicos y filogenéticos; así HINTON \& SERVICE (1969) lograron diferenciar los huevos de cuatro especies del género Aedes; SELIVON \& PERONDINI (1999) aportaron también evidencias para aclarar las relaciones taxonómicas y filogenéticas entre las especies de Anastrepha y ROZEN \& OZBEK (2005) sobre la filogenia de los géneros Anthophora y Thyreus.

En el presente trabajo nos proponemos ampliar el conocimiento de los aspectos excoriales del huevo de nueve especies incluidas en los géneros Triatoma, Meccus y Nesotriatoma, a través de un estudio cuantitativo y estructural de las formas poligonales de las celdas del exocorion. Por otra parte, de forma preliminar se describe matemáticamente a los arreglos poligonales de las celdas del exocorion con base en la teselación de superficies y se representan por medio de grafos.

\section{MATERIALES Y MÉTODOS}

Material entomológico. Los huevos proceden de parejas de ejemplares adultos de las especies: $T$. infestans (Klug), $T$. lenti (Sherlock \& Serafim), T. maculata, T. sordida (Stal), M. phyllosomus (Burmeister), M. picturatus Usinger, M. longipennis (Usinger), M. pallidipennis (Stal) y $N$. flavida (Neiva), provenientes de la colonia de triatominos del Laboratorio de Entomología "Herman Lent"; las parejas formadas fueron mantenidas bajo condiciones constantes de temperatura (28 ${ }^{\circ} \mathrm{C}$ ) y humedad relativa (50\%), estas parejas fueron alimentadas semanalmente hasta repleción con sangre de gallina Gallus domesticus (Linnaeus) mientras se colectaban los huevos, los cuales se colectaban diariamente y se mantenían bajo las mismas condiciones de humedad y temperatura hasta su eclosión. Fueron seleccionado aquellos huevos que no presentaban residuos ni deformaciones en su superficie.

Procesamiento de los huevos para la toma de micrografías. Los huevos seleccionados fueron lavados cuatro veces con agua destilada, el agua se removió con tiras de papel absorbente, luego se dejó secar el agua remanente colocando el vial durante dos días en una cámara de desecación con Sílicagel.

Los huevos se observaron en una lupa estereoscópica para seleccionar los que estaban libres de impurezas en la superficie, luego se colocaron en grupos de cuatro huevos por especie, en un soporte metálico empleando cinta doble adhesiva, los huevos fueron recubiertos con oro $100 \%$, empleando un cobertor iónico SPI Sputter Coater, hasta obtener una película de oro de aproximadamente $300 \AA \AA$ de espesor.

Toma de micrografías. Las micrografías se tomaron con un Microscopío Electrónico de Barrido Hitachi (S -2500).

Diseño del análisis. A cada región del huevo (cefálica, media y caudal) se le tomó una micrografía de 500x equivalente a una superficie de $385.000 \mu \mathrm{m}^{2}$ aproximadamente. Cada imagen fue dividida en 4 cuadrantes de aproximadamente $96.250 \mu \mathrm{m}^{2}$ cada uno, de tal modo que se dispuso de 4 réplicas (4 huevos) y 16 pseudoréplicas por región y por especie.

\section{Variables estimadas.}

Polígonos Totales (PT). Definido como la suma de todas las celdas que se les observaban todos sus lados completos.

Pentágonos (P). Definido como la suma de todas las celdas con cinco lados.

Hexágonos (Hx). Definido como la suma de todas las celdas con seis lados.

Heptágonos (Hp). Definido como la suma de todas las celdas con siete lados.

Segmentos Totales (ST). Definido como la suma de todos los segmentos.

En todos los casos, el número de polígonos o el número de segmentos, se expresan como el promedio de polígonos o segmentos en un área de $96.250 \mu \mathrm{m}^{2}$ de campo. La razón por la cual se estimaron pentágonos, hexágonos y heptágonos fue que éstos eran los comunes en todas las regiones y en todas las especies examinadas. La variable Polígonos Totales sin embargo, incluye tanto los comunes como los no comunes.

Teselaciones. Una teselación es un conjunto de tres o más polígonos los cuales comparten uno de sus vértices alrededor del cual los ángulos congruentes suman $360^{\circ}$; la teselación es denotada como la secuencia de números separados por puntos, donde cada número representa el número de lados de cada polígono que la conforma. Para denotar una teselación, el primer número corresponde al número de lados del polígono que primero aparece (completo o incompleto) en el cuadrante I de un plano dividido según se muestra en la Figura 1; el eje vertical de este plano coincide con el eje longitudinal cefálico-caudal del huevo. Los siguientes números de la denotación corresponden al número de lados de los polígonos que aparecen en dicho plano en sentido horario. Las teselaciones fueron extraídas según criterio mostrado en la Figura 2. Se elaboró un catálogo de las teselaciones observadas en un campo de $385.000 \mu^{2}$, es decir, el catálogo se elaboró con base en 108 campos de la superficie del huevo. 
Las teselaciones observadas se agruparon como compartidas y no compartidas. Como compartidas definimos aquellas teselaciones que aparecieron en los 108 campos. Como no compartidas definimos aquellas teselaciones que no aparecieron en los 108 campos. En ambas se encontraron teselaciones formadas por tres polígonos con tres o dos tipos de polígonos o un sólo tipo de polígono, pero sólo en las teselaciones no compartidas se encontraron teselaciones formadas por cuatro polígonos, en las cuales habían con tres o dos tipos de polígonos o un sólo tipo de polígono. Finalmente en éstas también se encontraron teselaciones agregadas, las cuales se definen como dos teselaciones constituidas por 4 polígonos cada una intercaladas por una o más teselaciones de tres polígonos. Además, por agregada simétrica se define aquella teselación que se dispone de forma simétrica alrededor del eje cefálico-caudal, y como agregada asimétrica la que no se dispone simétricamente alrededor de dicho eje.

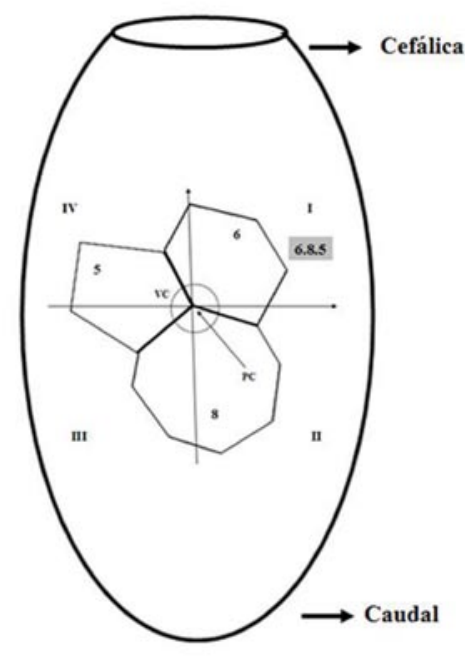

Figura 1. Ejemplo de cómo se denotó una teselación. En el cuadrante I de un plano cuyo eje vertical coincide con el eje cefálico-caudal del huevo, aparece en el sentido horario, primero un polígono de 6 lados, luego uno de 8 lados y finalmente otro de 5 lados, obteniéndose así una teselación 6.8.5. PC: punto central, VC: vértices congruentes.

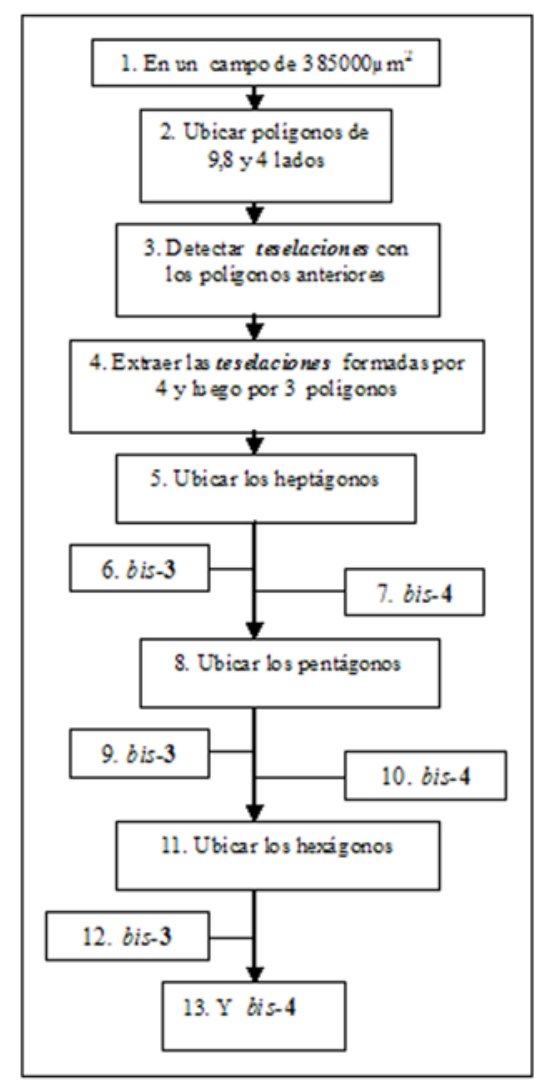

Figura 2. Protocolo de extracción de teselaciones en una micrografía.
Grafos. Sea V un conjunto no vacío. Por [V] ${ }^{\mathrm{k}}$ se denotará el conjunto de todos los subconjuntos de k elementos de V. Un grafo es un par $\mathrm{G}=(\mathrm{V}, \mathrm{A})$ de conjuntos tales que $\mathrm{A} \subseteq[\mathrm{V}]^{2}$; esto es, los elementos de A son subconjuntos de 2 elementos de V. Los elementos de $\mathrm{V}$ son los vértices (o nodos, o puntos) del grafo G, los elementos de A son sus aristas (o arcos). La manera usual de representar un grafo es dibujar un punto por cada vértice y unir dos de esos puntos por una línea si los correspondientes vértices forman una arista (DIESTEL 2005).

Análisis estadístico. Para cada variable analizada (PT, P, Hx, Hp y ST) se aplicó la prueba de Kolmogorov-Smirnov para conocer si los datos procedían de poblaciones de datos distribuidas normalmente. Dado que sólo algunas variables presentaron esta distribución, se decidió usar el equivalente no paramétrico del análisis de varianza, la prueba de KruskalWallis, en todas las variables analizadas. Se tomó como nivel estadístico de significación "p" o,05. Los análisis estadísticos fueron llevados a cabo usando SPSS 10.0 (Statistical Product and Service Solutions).

\section{RESULTADOS}

Polígonos Totales (PT). Los promedios de PT por campo en el huevo completo variaron desde $12,90 \pm 8,84$ en $M$. picturatus hasta 26,46 \pm 4,26 en $T$. infestans (ver Tabla 1). En la región cefálica los promedios de PT variaron desde 14,38 \pm 2,78 en $M$. phyllosomus hasta 29,00 \pm 4,82 en $T$. infestans. En la región media los promedios de PT variaron desde $11,81 \pm 2,56$ en $M$. picturatus hasta $24,56 \pm 3,41$ en $T$. infestans. En la región caudal los promedios de PT variaron desde 10,81 $\pm 2,23$ en $M$. picturatus hasta $25,81 \pm 3,29$ en $T$. infestans. Se encontraron diferencias estadísticamente significativas entre los promedios de PT del huevo completo de las especies examinadas $(\mathrm{p}<0,05)$. Cuando se analizaron los promedios de PT por regiones del huevo, se encontraron diferencias estadísticamente significativas por región entre todas las especies examinadas $(\mathrm{p}<0,05)$. De igual forma se encontró que todas las especies difieren significativamente $(\mathrm{p}<\mathrm{0,05}$ ) en el promedio de PT entre regiones (ver Tablas 2, 3 y 4). La prueba no paramétrica de U-Mann-Whitney reveló que no hay diferencias estadísticamente significativas ( $p>0,05)$ en el promedio de PT en el huevo en los siguientes grupos de similaridad T. maculata - T. sordida y $M$. phyllosomus $-M$. picturatus $-M$. longipennis.

Pentágonos (P). Los promedios de P por campo en el huevo completo variaron desde 2,29 $\pm 1,34$ en $M$. picturatus hasta 4,52 $\pm 1,84$ en $T$. lenti (ver Tabla 1). En la región cefálica los promedios de $\mathrm{P}$ variaron desde 2,88 $\pm 1,50$ en $N$. flavida hasta 5,56 $\pm 2,31$ en $T$. lenti. En la región media los promedios de $\mathrm{P}$ variaron desde $1,88 \pm 1,26$ en $M$. picturatus hasta $4,31 \pm 1,58$ en $T$. lenti. En la región caudal los promedios de $\mathrm{P}$ variaron desde $1,75 \pm 1$, oo en M. phyllosomus hasta 4,69 $\pm 2,12$ en $T$. infestans. Se encontraron diferencias estadísticamente significativas entre los promedios de $\mathrm{P}$ del huevo completo de las especies examinadas $(\mathrm{p}<0,05)$. Cuando se analizaron los promedios de $\mathrm{P}$ por regiones del huevo, se encontraron diferencias estadísticamente significativas entre todas las especies examinadas $(\mathrm{p}<0,05)$. De igual forma se encontró que todas las especies difieren significativamente $(\mathrm{p}<\mathrm{0}, 05)$ en el promedio de $\mathrm{P}$ entre regiones (ver Tablas 2, 3 y 4). La prueba no paramétrica de U-Mann-Whitney reveló que no hay diferencias estadísticamente significativas ( $p>0,05$ ) en el promedio de $\mathrm{P}$ en el huevo en los siguientes grupos de similaridad T. infestans $-T$. lenti $-T$. sordida $-T$. pallidipennis, $T$. maculata $-M$. longipennis $-M$. pallidipennis $-N$. flavida $\mathrm{y}$ $M$. phyllosomus $-M$. picturatus $-M$. longipennis $-N$. flavida.

Hexágonos (Hx). Los promedios de $\mathrm{Hx}$ por campo en el huevo completo variaron desde $8,48 \pm 4,13$ en $M$. picturatus hasta $19,15 \pm 4,18$ en $T$. infestans (ver Tabla 1). En la región 
Tabla 1. Promedio de Polígonos Totales (PT), Pentágonos (P), Hexágonos (Hx), Heptágonos (Hp) y Segmentos Totales (ST) del huevo.

\begin{tabular}{|c|c|c|c|c|c|}
\hline Especie & $\begin{array}{c}\text { PT } \\
(\mathrm{X} \pm \mathrm{SD})\end{array}$ & $\begin{array}{c}P \\
(X \pm S D)\end{array}$ & $\begin{array}{c}\mathbf{H x} \\
(X \pm S D)\end{array}$ & $\begin{array}{c}\text { Hp } \\
(X \pm S D)\end{array}$ & $\begin{array}{c}\text { ST } \\
\left(X_{ \pm} \text {SD }\right)\end{array}$ \\
\hline T. infestans & $26,46 \pm 4,26$ & $4,38 \pm 2,59$ & $19,15 \pm 4,18$ & $2,63 \pm 1,27$ & $117,90 \pm 14,65$ \\
\hline T. lenti & $23,19 \pm 4,22$ & $4,52 \pm 1,84$ & $15,94 \pm 3,86$ & $2,58 \pm 1,25$ & $105,13 \pm 14,56$ \\
\hline T. maculata & $20,92 \pm 4,03$ & $3,17 \pm 1,59$ & $15,75 \pm 3,80$ & $1,81 \pm 1,23$ & $96,85 \pm 13,61$ \\
\hline T. sordida & $19,54 \pm 2,94$ & $4,06 \pm 1,94$ & $13,44 \pm 3,18$ & $1,77 \pm 1,15$ & $92,23 \pm 9,45$ \\
\hline M. phyllosomus & $13,27 \pm 2,53$ & $2,44 \pm 1,29$ & $8,88 \pm 2,38$ & $1,58 \pm 1,01$ & $69,25 \pm 14,72$ \\
\hline M. picturatus & $12,90 \pm 4,84$ & $2,29 \pm 1,34$ & $8,48 \pm 4,13$ & $1,88 \pm 1,20$ & $68,42 \pm 16,21$ \\
\hline M. longipennis & $13,69 \pm 2,72$ & $2,73 \pm 1,66$ & $9,27 \pm 2,83$ & $1,60 \pm 1,25$ & $69,04 \pm 8,63$ \\
\hline M. pallidipennis & $17,42 \pm 3,49$ & $3,60 \pm 1,70$ & $11,25 \pm 3,27$ & $2,17 \pm 1,31$ & $86,33 \pm 13,43$ \\
\hline N. flavida & $19,25 \pm 3,99$ & $2,77 \pm 1,39$ & $15,17 \pm 3,84$ & $1,46 \pm 1,11$ & $91,25 \pm 14,97$ \\
\hline
\end{tabular}

Tabla 2. Promedio de Polígonos Totales (PT), Pentágonos (P), Hexágonos (Hx), Heptágonos (Hp) y Segmentos Totales (ST) en la región cefálica del huevo.

\begin{tabular}{|c|c|c|c|c|c|}
\hline \multirow[b]{2}{*}{ Especies } & \multicolumn{5}{|c|}{ Cefálica } \\
\hline & $\begin{array}{c}\text { PT } \\
(\mathrm{X} \pm \mathrm{SD}) \\
\end{array}$ & $\begin{array}{c}P \\
(X \pm S D)\end{array}$ & $\begin{array}{c}\mathbf{H x} \\
(\mathrm{X} \pm \mathrm{SD}) \\
\end{array}$ & $\begin{array}{c}\text { Hp } \\
(\mathrm{X} \pm \mathrm{SD})\end{array}$ & $\begin{array}{c}\text { ST } \\
(\mathbf{X} \pm \mathbf{S D}) \\
\end{array}$ \\
\hline T. infestans & $29,06 \pm 4,82$ & $5,50 \pm 3,16$ & $20,00 \pm 5,19$ & $2,44 \pm 1,09$ & $126,00 \pm 18,42$ \\
\hline T. lenti & $25,56 \pm 4,79$ & $5,56 \pm 2,31$ & $16,88 \pm 4,03$ & $2,88 \pm 1,41$ & $115,25 \pm 15,29$ \\
\hline T. maculata & $23,19 \pm 6,00$ & $3,63 \pm 1,82$ & $17,75 \pm 4,60$ & $1,63 \pm 1,02$ & $106,13 \pm 19,55$ \\
\hline T. sordida & $21,25 \pm 3,45$ & $4,31 \pm 1,49$ & $15,38 \pm 3,18$ & $1,50 \pm 0,89$ & $98,75 \pm 9,40$ \\
\hline M. phyllosomus & $14,38 \pm 2,78$ & $3,44 \pm 1,31$ & $8,56 \pm 2,83$ & $1,81 \pm 0,75$ & $75,56 \pm 13,15$ \\
\hline M. picturatus & $16,06 \pm 6,74$ & $2,88 \pm 1,67$ & $10,94 \pm 4,52$ & $2,25 \pm 1,34$ & $78,94 \pm 23,68$ \\
\hline M. longipennis & $15,06 \pm 3,99$ & $2,88 \pm 1,54$ & $10,31 \pm 3,59$ & $1,75 \pm 1,48$ & $72,63 \pm 13,50$ \\
\hline M. pallidipennis & $20,38 \pm 4,57$ & $4,31 \pm 1,81$ & $12,69 \pm 4,32$ & $2,94 \pm 1,48$ & $98,44 \pm 16,92$ \\
\hline N. flavida & $22,63 \pm 5,16$ & $2,88 \pm 1,50$ & $18,31 \pm 4,39$ & $1,25 \pm 1,00$ & $104,81 \pm 19,20$ \\
\hline
\end{tabular}

Tabla 3. Promedio de Polígonos Totales (PT), Pentágonos (P), Hexágonos (Hx), Heptágonos (Hp) y Segmentos Totales (ST) en la región media del huevo.

\begin{tabular}{|c|c|c|c|c|c|}
\hline \multirow[b]{2}{*}{ Especies } & \multicolumn{5}{|c|}{ Media } \\
\hline & $\begin{array}{c}\mathrm{PT} \\
(\mathrm{X} \pm \mathrm{SD})\end{array}$ & $\begin{array}{c}P \\
(X \pm S D)\end{array}$ & $\begin{array}{c}\mathrm{Hx} \\
(\mathrm{X} \pm \mathrm{SD})\end{array}$ & $\begin{array}{c}\mathrm{Hp} \\
(\mathrm{X} \pm \mathrm{SD})\end{array}$ & $\mathrm{ST}(\mathrm{X} \pm \mathrm{SD})$ \\
\hline T. infestans & $24,56 \pm 3,141$ & $2,94 \pm 1,69$ & $19,31 \pm 3,94$ & $2,13 \pm 1,09$ & $111,94 \pm 10,27$ \\
\hline T. lenti & $21,31 \pm 3,48$ & $4,31 \pm 1,58$ & $14,31 \pm 3,84$ & $2,56 \pm 0,96$ & $96,75^{ \pm 11,80}$ \\
\hline T. maculata & $19,25 \pm 1,84$ & $2,69 \pm 1,30$ & $14,69 \pm 2,18$ & $1,75 \pm 1,34$ & $90,75 \pm 5,47$ \\
\hline T. sordida & $18,06 \pm 1,77$ & $3,56 \pm 2,06$ & $12,25 \pm 2,27$ & $1,94 \pm 1,24$ & $87,19 \pm 6,26$ \\
\hline M. phyllosomus & $12,63 \pm 2,42$ & $2,50 \pm 1,46$ & $8,75 \pm 2,52$ & $1,50 \pm 1,03$ & $67,81 \pm 9,40$ \\
\hline M. picturatus & $11,81 \pm 2,56$ & $1,88 \pm 1,26$ & $8,56 \pm 3,63$ & $1,31 \pm 1,08$ & $64,38 \pm 6,40$ \\
\hline M. longipennis & $13,06 \pm 1,48$ & $2,94 \pm 1,65$ & $8,44 \pm 2,10$ & $1,56 \pm 1,26$ & $67,25 \pm 4,02$ \\
\hline M. pallidipennis & $16,27 \pm 1,16$ & $3,33 \pm 1,72$ & $10,80 \pm 1,78$ & $1,87 \pm 1,19$ & $79,47 \pm 4,72$ \\
\hline N. flavida & $16,82 \pm 1,63$ & $2,41 \pm 1,18$ & $13,24 \pm 2,14$ & $1,47 \pm 0,94$ & $82,29 \pm 3,92$ \\
\hline
\end{tabular}

Tabla 4. Promedio de Polígonos Totales (PT), Pentágonos (P), Hexágonos (Hx), Heptágonos (Hp) y Segmentos Totales (ST) en la región caudal del huevo.

\begin{tabular}{|c|c|c|c|c|c|}
\hline \multirow{2}{*}{ Especies } & \multicolumn{5}{|c|}{ Caudal } \\
\hline & $\mathrm{PT}(\mathrm{X} \pm \mathrm{SD})$ & $\mathbf{P}(\mathrm{X} \pm \mathrm{SD})$ & $\mathrm{Hx}(\mathrm{X} \pm \mathrm{SD})$ & $\mathrm{Hp}(\mathrm{X} \pm \mathrm{SD})$ & $\mathrm{ST}(\mathrm{X} \pm \mathrm{SD})$ \\
\hline T. infestans & $25,81 \pm 3,29$ & $4,69 \pm 2,12$ & $18,13 \pm 3,20$ & $3,31 \pm 1,35$ & $115,75 \pm 10,71$ \\
\hline T. lenti & $22,69 \pm 3,28$ & $3,69 \pm 0,95$ & $16,63 \pm 3,38$ & $2,31 \pm 1,35$ & $103,13 \pm 10,42$ \\
\hline T. maculata & $20,31 \pm 1,58$ & $3,19 \pm 1,56$ & $14,63 \pm 3,72$ & $2,06 \pm 1,34$ & $93,56 \pm 5,37$ \\
\hline T. sordida & $19,31 \pm 2,55$ & $4,19 \pm 2,34$ & $12,69 \pm 3,22$ & $1,88 \pm 1,31$ & $90,75 \pm 8,80$ \\
\hline M. phyllosomus & $10,81 \pm 2,14$ & $1,75 \pm 1, \mathrm{Oo}$ & $9,31 \pm 1,78$ & $1,44 \pm 1,21$ & $68,56 \pm 7,11$ \\
\hline M. picturatus & $10,81 \pm 2,23$ & $2,13 \pm 0,81$ & $6,50 \pm 2,68$ & $2,06 \pm 1,00$ & $61,94 \pm 6,42$ \\
\hline M. longipennis & $13,13 \pm 1,50$ & $2,38 \pm 1,82$ & $9,06 \pm 2,41$ & $1,50 \pm 1,03$ & $67,25 \pm 3,64$ \\
\hline M. pallidipennis & $15,69 \pm 1,30$ & $3,31 \pm 1,35$ & $10,06 \pm 2,59$ & $1,81 \pm 0,83$ & $81,38 \pm 4,44$ \\
\hline
\end{tabular}


cefálica los promedios de Hx variaron desde 8,56 \pm 2,83 en M. phyllosoma hasta 20,00 $\pm 5,19$ en $T$. infestans. En la región media los promedios de Hx variaron desde 8,44 $\pm 2,10$ en $M$. longipennis hasta $19,31 \pm 3,94$ en $T$. infestans. En la región caudal los promedios de $\mathrm{Hx}$ variaron desde 6,50 \pm 2,68 en $M$. picturatus hasta $18,13 \pm 3,20$ en $T$. infestans. Se encontraron diferencias estadísticamente significativas entre los promedios de Hx del huevo completo de las especies examinadas $(\mathrm{p}<0,05)$. Cuando se analizaron los promedios de $\mathrm{Hx}$ por regiones del huevo, se encontraron diferencias estadísticamente significativas entre todas las especies examinadas $(\mathrm{p}<0,05)$. De igual forma se encontró que todas las especies difieren significativamente $(\mathrm{p}<\mathrm{0,05}$ ) en el promedio de $\mathrm{Hx}$ entre regiones. La prueba no paramétrica de U-Mann-Whitney reveló que no hay diferencias estadísticamente significativas ( $\mathrm{p}>0,05)$ en el promedio de $\mathrm{Hx}$ en el huevo en los siguientes grupos de similaridad T. lenti $-T$. maculata - N. flavida y $M$. phyllosomus $-M$. picturatus $-M$. longipennis.

Heptágonos (Hp). Los promedios de Hp por campo en el huevo completo variaron desde $1,46 \pm 1,11$ en $N$. flavida hasta $2,63 \pm 1,27$ en $T$. infestans (ver Tabla 1). En la región cefálica los promedios de $\mathrm{Hp}$ variaron desde 1,25 \pm 1,00 en $N$. flavida hasta $2,94 \pm 1,48$ en $M$. pallidipennis. En la región media los promedios de $\mathrm{Hp}$ variaron desde $1,31 \pm 1,08$ en $M$. picturatus hasta 2,56 0,96 en T. lenti. En la región caudal los promedios de Hp variaron desde 1,44 \pm 1,21 en M. phyllosomus hasta 3,31 \pm 1,35 en $T$. infestans. Se encontraron diferencias estadísticamente significativas entre los promedios de $\mathrm{Hp}$ del huevo completo de todas las especies examinadas $(\mathrm{p}<0,05)$. Cuando se analizaron los promedios de $\mathrm{Hp}$ por regiones del huevo, se encontraron diferencias estadísticamente significativas entre las regiones caudales de todas las especies $(\mathrm{p}<0,05)$ y entre las regiones cefálicas de todas las especies $(\mathrm{p}<0,05)$, mientras que entre las regiones medias de todas las especies no se encontraron diferencias estadísticamente significativas $(p>0,05)$. La prueba no paramétrica de U-Mann-Whitney reveló que no hay diferencias estadísticamente significativas ( $\mathrm{p}>0,05$ ) en el promedio de $\mathrm{Hp}$ en el huevo en los siguientes grupos de similaridad T. infestans - M. pallidipennis, M. pallidipennis - T. lenti y T. maculata $-T$. sordida $-M$. phyllosomus - M. picturatus - M. longipennis - M. pallidipennis - N. flavida.

Segmentos Totales (ST). Los promedios de ST por campo en el huevo completo variaron desde $68,42 \pm 16,21$ en $M$. picturatus hasta 117,90 $\pm 14,62$ en T. infestans (ver Tabla 1). En la región cefálica los promedios de ST variaron desde 72,63 \pm 13,50 en $M$. longipennis hasta $126,00 \pm 18,42$ en $T$. infestans. En la región media los promedios de ST variaron desde 64,38 $\pm 6,40$ en $M$. picturatus hasta $111,94 \pm 10,27$ en $T$. infestans. En la región caudal los promedios de ST variaron desde 61,94 $\pm 6,42$ en $M$. picturatus hasta $115,75 \pm 10,71$ en $T$. infestans. Cuando se analizaron los promedios de ST por regiones del huevo, se encontraron diferencias estadísticamente significativas entre todas las especies examinadas $(\mathrm{p}<0,05)$ De igual forma se encontró que todas las especies difieren significativamente $(\mathrm{p}<\mathrm{0,05})$ en el promedio de ST entre regiones (ver Tablas 2, 3 y 4). La prueba no paramétrica de U-Mann-Whitney reveló que no hay diferencias estadísticamente significativas ( $p>0,05)$ en el promedio de ST en el huevo en los siguientes grupos de similaridad T. maculata - T. sordida, T. sordida - N. flavida y $M$. phyllosomus $-M$. picturatus $-M$. longipennis.

En resumen podemos decir que tanto la región cefálica como caudal, son diferentes en todas las especies examinadas en cuanto a todos los caracteres estudiados, mientras que la región media es diferente en todas las especies examinadas sólo en cuanto a los caracteres PT, P, Hx y ST, es decir las especies son iguales en cuanto a los heptágonos de la región media. Por otra parte, las regiones cefálica, media y caudal al ser comparadas en una misma especie, son diferentes entre si en cuanto sólo a los caracteres PT, P, Hx y ST, es decir, las regiones son iguales en una misma especie sólo en cuanto a los heptágonos.

Teselaciones. Entre las teselaciones compartidas sólo se encontraron teselaciones con 3 polígonos, en un caso 2 tipos de polígonos y en otro con un sólo tipo de polígono. Por otra parte, se encontró una variedad mayor de teselaciones no compartidas, entre las cuales se observaron con 3 y 4 tipos de polígonos. Las teselaciones de 3 polígonos incluían con 3 y 2 tipos de polígonos diferentes. Las teselaciones de 4 polígonos incluían con 3 o 2 tipos de polígonos y 1 tipo de polígono. Entre las no compartidas se encontraron también las teselaciones de tipo aglomeradas, las cuales incluían teselaciones que presentaban simetría y otras sin simetría.

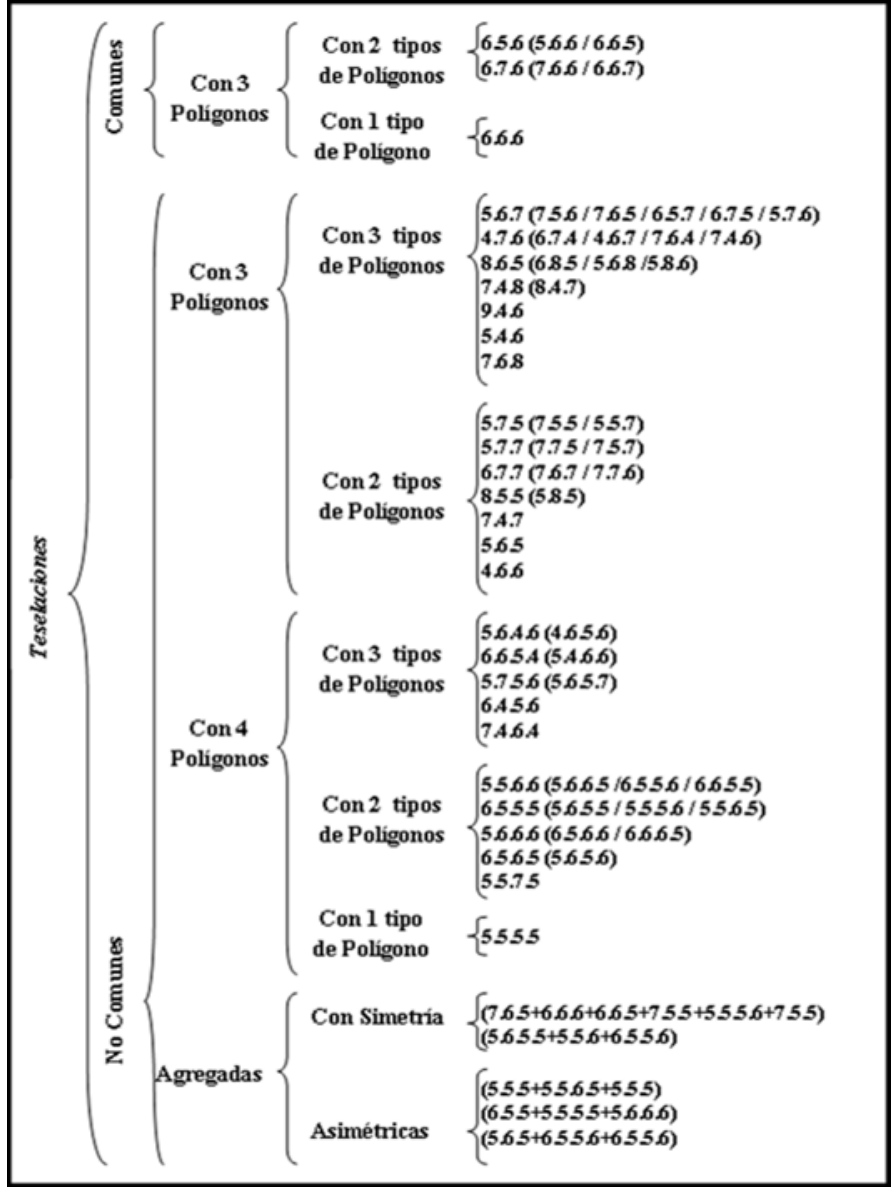

Figura 3. Variedad de teselaciones encontradas en los 108 campos de $385.000 \mu \mathrm{m}^{2} \mathrm{c} / \mathrm{u}$. que incluyen a todas las especies examinadas.

En las Figs. 4-6 se muestran las variedades de teselaciones encontradas en los 108 campos de $385.000 \mu \mathrm{m}^{2}$ cada uno, destacando que las teselaciones mostradas por especie, sólo indican que dicha teselación es un ejemplo de las encontradas en esa especie, y no necesariamente una de las teselaciones no pueda ser encontrada en varias especies. Las teselaciones extraídas y mostradas en la Figs. 4-6 fueron representadas mediante grafos, estos grafos se muestran en las Tablas 5-11.

\section{DISCUSIÓN}

Es un hecho bien conocido que el esculpido del exocorion de los triatominos es una herramienta taxonómica. Así, en el género Triatoma se ha encontrado que caracteres como la forma, la regularidad y el número de lados de los polígonos que constituyen las celdas, se han encontrado que varían según la especie (BRICEÑO-IRAGORRY 1934; BARTH \& MUTH 1958; LENT \& WYGODZINSKY 1979; BARATA 1981,1998; GONCALVES et al. 1985; SILVA et al. 2005; VISCIARELLI et al. 2004) e incluso entre poblaciones alopátricas (COSTA et al.1997). Por otra parte, especies que se agrupaban en el género Triatoma y que GALVÃO et al. (2003) recientemente agrupa en el género Meccus, OBARA et al. (2007) valida este agrupamiento al encontrar que tres de las cuatro especies estudiadas en este género, se asemejan en la arquitectura del exocorion. 


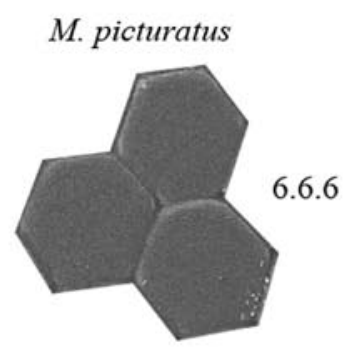

T. lenti

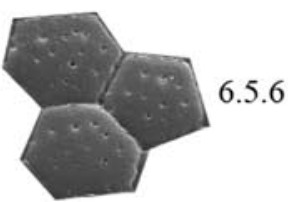

T. infestans
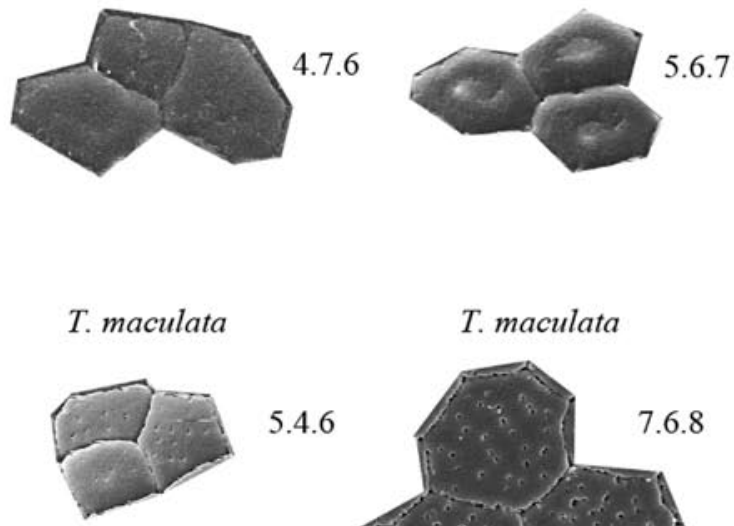

5.4 .6

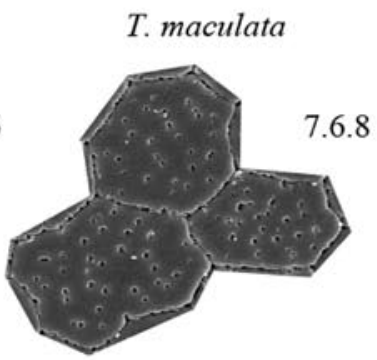

M. pallidipennis

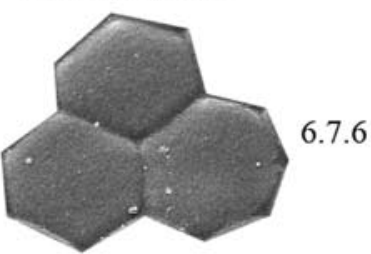

M. longipennis

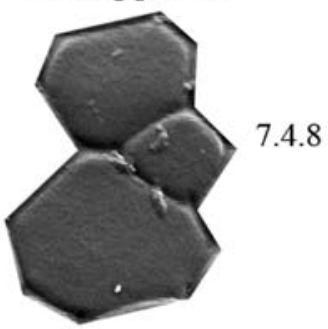

M. pallidipennis

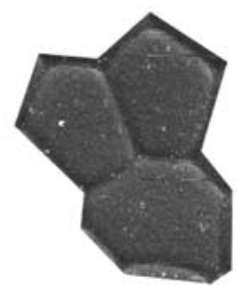

5.7 .5
T. maculata

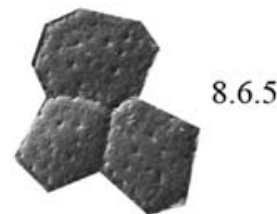

T. lenti
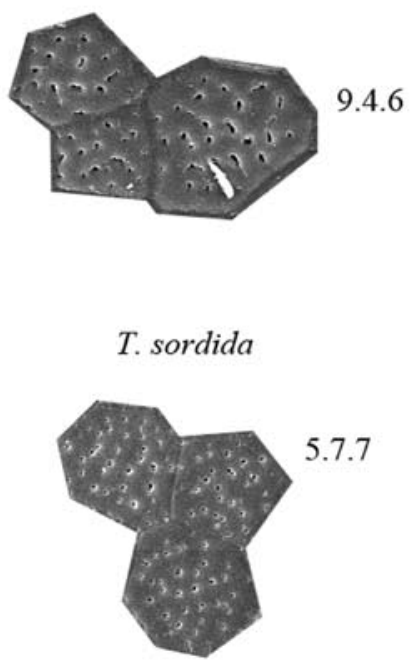

Figura 4. Ejemplos de teselaciones observadas en las especies M. picturatus, M. pallidipennis, M. longipennis, T. maculata, T. lenti, T. sordida y T. infestans.
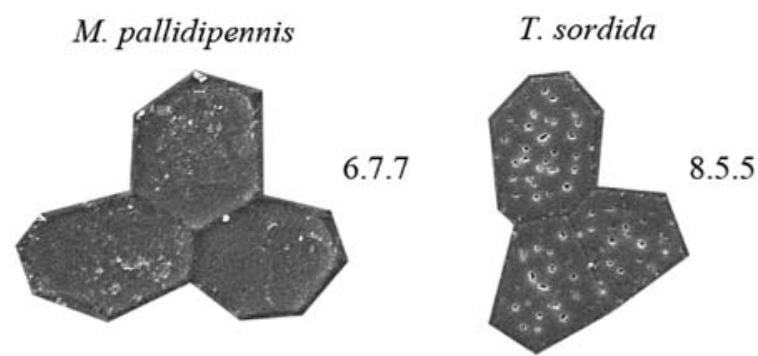

T. infestans

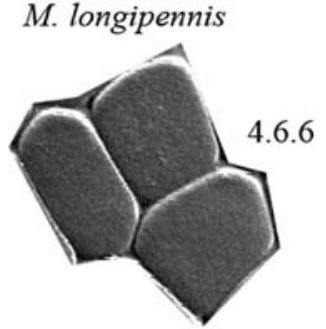

T. lenti

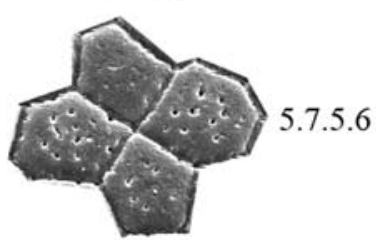

T. lenti

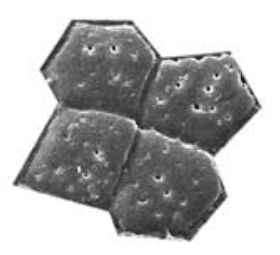

T. lenti

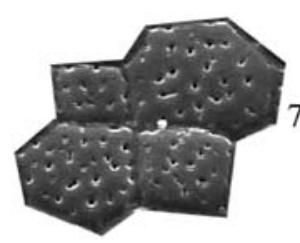

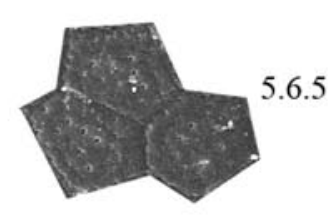

T. sordida

5.6.4.6

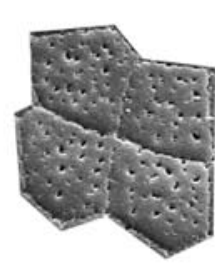

6.4.5.6

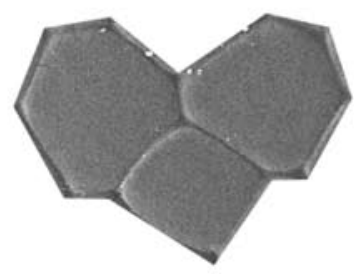

7.4.7

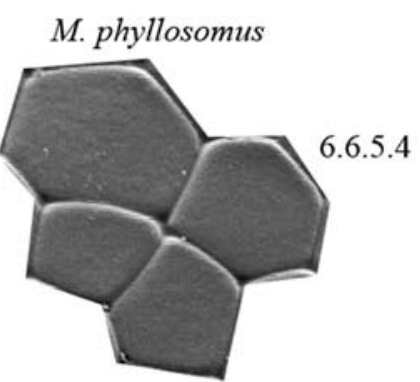

M. longipennis

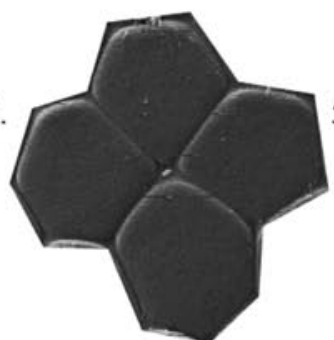

T. infestans

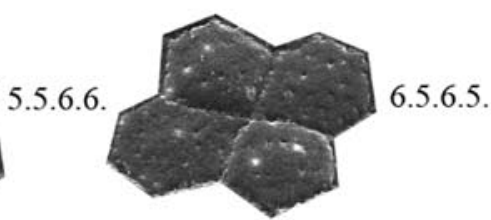

Figura 5. Ejemplos de teselaciones observadas en las especies M. phyllosomus, M. picturatus, M. pallidipennis, M. longipennis, T. lenti, T. sordida y T. infestans. 

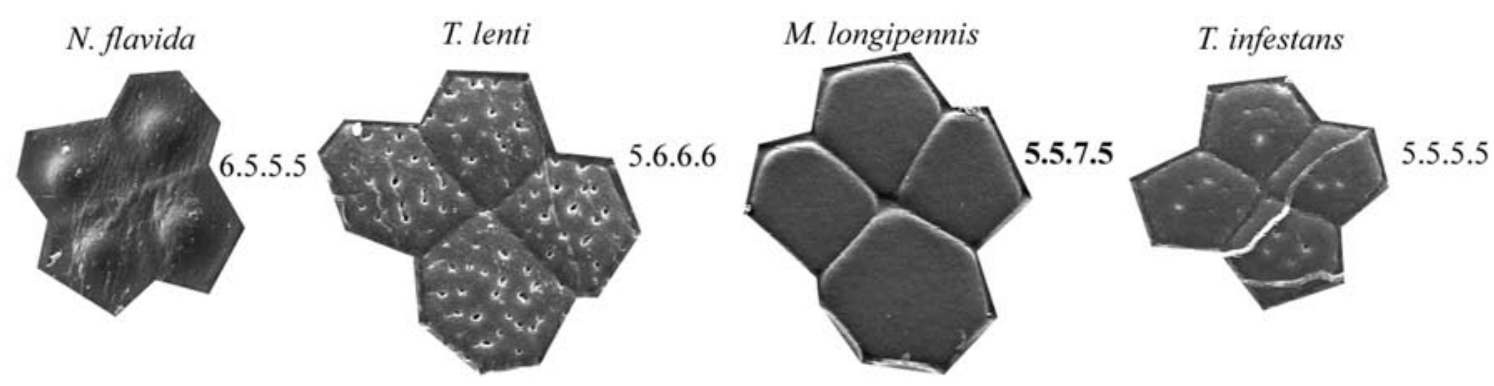

T. lenti
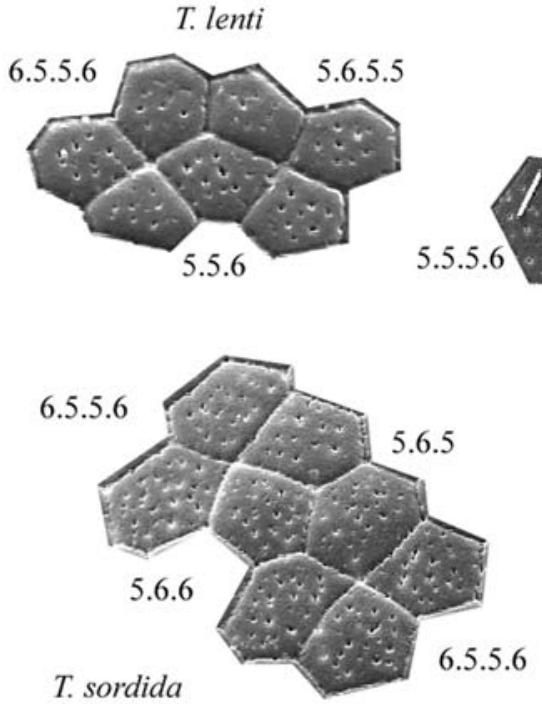

T. sordida
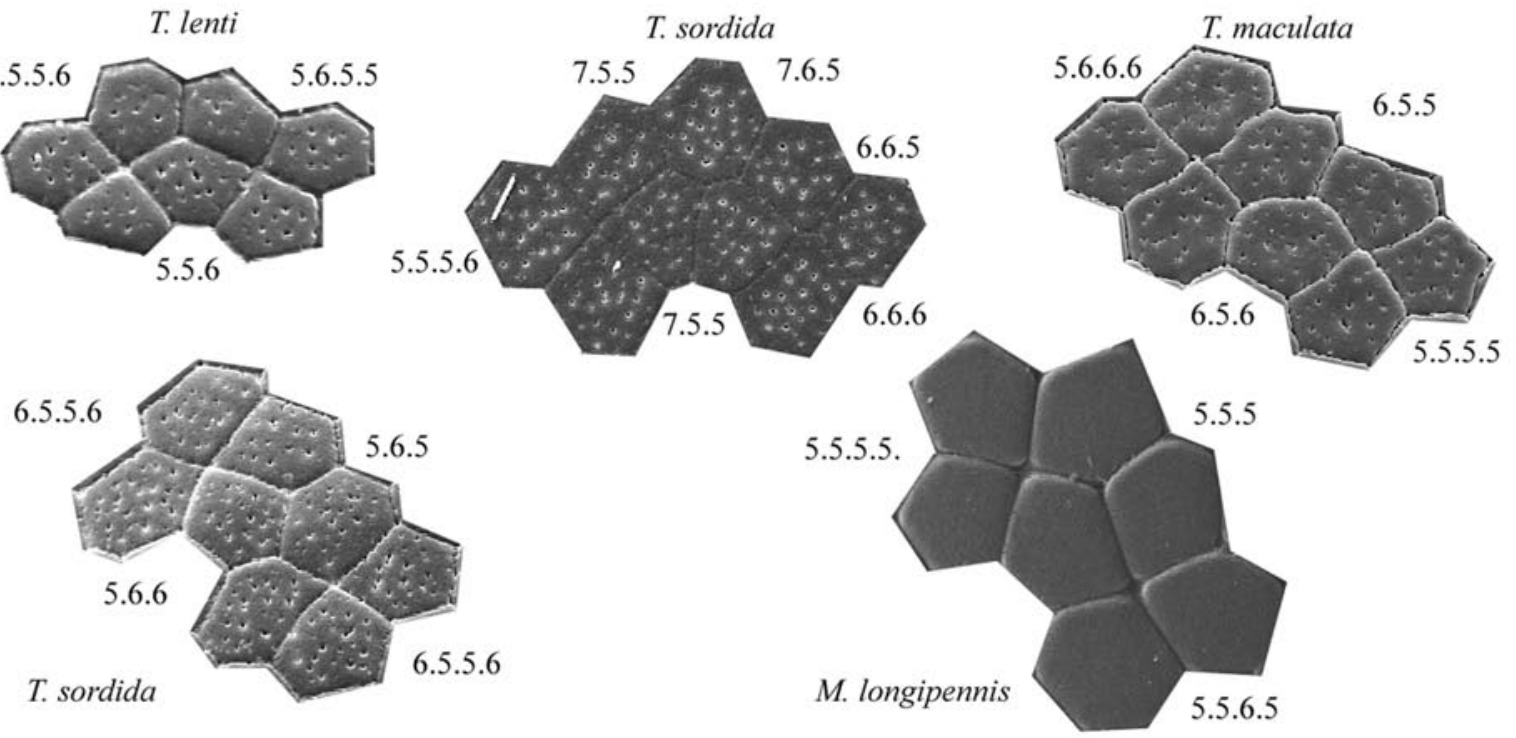

Figura 6. Ejemplos de teselaciones observadas en las especies M. longipennis, T. lenti, T. sordida, T. infestans T. maculata y N. flavida.

En los estudios mencionados, el análisis de la superficie del exocorion no distingue entre las regiones del huevo, excepto el de VISCIARELLI et al. (2004), en el cual se analizan cualitativamente y por separado las regiones cefálica, media y caudal de $T$. patagonica; es decir, los estudios de la arquitectura del exocorion generalmente no han abordado aspectos geométricos tales como las proporciones de las celdas poligonales y sus arreglos en el mosaico exocorial.

En el presente trabajo se estudió de forma cuantitativa y estructural las celdas exocoriales en huevos eclosionados de nueve especies incluidas en los géneros Triatoma, Nesotriatoma y Meccus; encontrándose entre los polígonos comunes a pentágonos, hexágonos y heptágonos, siendo el hexágono el más abundante, seguido del pentágono y luego del heptágono en la totalidad del huevo. También se encontró que los promedios del número de pentágonos y hexágonos son estadísticamente diferentes al comparar la misma región en diferentes especies y las diferentes regiones en una misma especie, mientras que el número promedio de heptágonos fue diferente sólo al comparar la región cefálica o caudal de diferentes especies, es decir, el promedio de heptágonos no varía al comparar la región media de las diferentes especies. Por otra parte, el número promedio de heptágonos no varió al comparar las diferentes regiones en una misma especie.

El hecho que el promedio de heptágonos no haya variado entre las regiones de una misma especie, lo atribuimos a que este polígono por poseer mayor número de lados que el pentágono y el hexágono, es más deformable y por tanto no se impone una restricción a su frecuencia según la región del huevo.

Por otra parte se encontró que el promedio de hexágonos, pentágonos, polígonos totales y segmentos totales fueron similares en las cuatro especies estudiadas del género Meccus, lo cual valida el agrupamiento propuesto por GALVÃO et al. (2003) de estas especies en este género antes incluidas en el género Triatoma; ampliándose así los caracteres que permiten la identificación del género Meccus.
PRESTON (1969) estudiando los huevos de varias familias de aves de Norteamérica, encontró que las siluetas de los huevos son distintas en tamaño, pudiendo ser caracterizadas específicamente por tres parámetros como son: el alargamiento, la asimetría y el bicono. Con estos tres parámetros o especificadores de la forma, este autor considera que se podría definir con exactitud el contorno de cualquier huevo de ave. La forma ovoide bien sea del huevo de un ave o de un triatomino, estaría por tanto regida por estos especificadores de la silueta. Por otra parte, más recientemente, CASTRO et al. (1998) y TAMASSIA \& TOLLIS (1989), estudiaron las teselaciones de un grafo en superficie; estos autores asocian a un grafo una partición en baldosas de una superficie (plana o no plana), utilizando como baldosas rectángulos (degenerados o no). En el presente trabajo, tratamos el problema de forma inversa, es decir, extrajimos las teselaciones del exocorion de un huevo de triatomino y luego las representamos por medio de grafos.

En virtud de lo dicho, consideramos que los polígonos que constituyen la estructura de una superficie curva de un ovoide como lo es el huevo de un triatomino, está regido por unos pocos principios geométricos. Tales principios estarían limitados por factores como la invariable función del huevo (adaptativofuncional), la variedad de la forma del huevo en un grupo de especies más estrechamente relacionadas filogenéticamente (histórico-filogenético) y el proceso de formación del exocorion (morfogenético-construccional). Consideramos por consiguiente, que la evolución de las variedades de la forma ovoide de los huevos de los triatominos, son el producto de la relación dinámica de estos factores.

El protocolo de análisis de los polígonos del exocorion mediante las teselaciones y su representación por medio de grafos, propuesto en este trabajo, constituye una aproximación preliminar de futuros estudios que permitirán el hallazgo y comprensión de los principios geométricos a los que están sujetos los arreglos de las celdas exocoriales. 

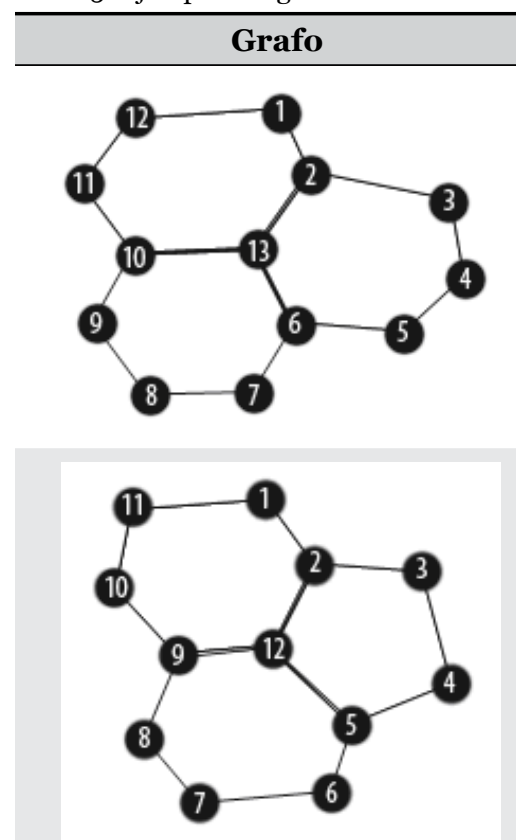

$$
\begin{gathered}
\mathrm{G}_{666}=\left(\mathrm{V}_{666}, \mathrm{~A}_{666}\right) \\
\text { T. infestans }
\end{gathered}
$$

$\mathrm{V}_{666}=\{1, \ldots \ldots ., 13\}$

$\mathrm{A}_{666}=\{\{1,2\},\{2,3\},\{3,4\},\{4,5\},\{5,6\},\{6,7\}$, $\{7,8\},\{8,9\},\{9,10\},\{10,11\},\{11,12\},\{12,1\}$, $\{2,13\},\{6,13\},\{10,13\}\}$

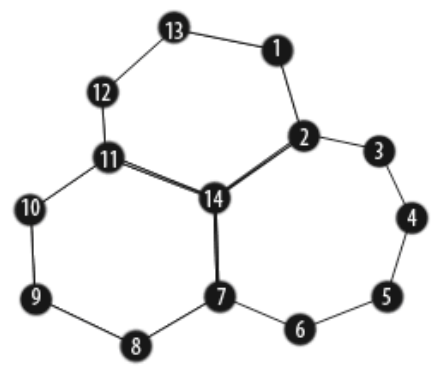

$$
\begin{gathered}
\mathrm{G}_{656}=\left(\mathrm{V}_{656}, \mathrm{~A}_{656}\right) \\
\text { T. lenti }
\end{gathered}
$$$$
\mathrm{V}_{656}=\{1, \ldots \ldots, 12\}
$$

$\mathrm{A}_{656}=\{\{1,2\},\{2,3\},\{3,4\},\{4,5\},\{5,6\},\{6,7\}$, $\{7,8\},\{8,9\},\{9,10\},\{10,11\},\{11,1\},\{2,12\}$, $\{5,12\},\{6,13\},\{9,12\}\}$

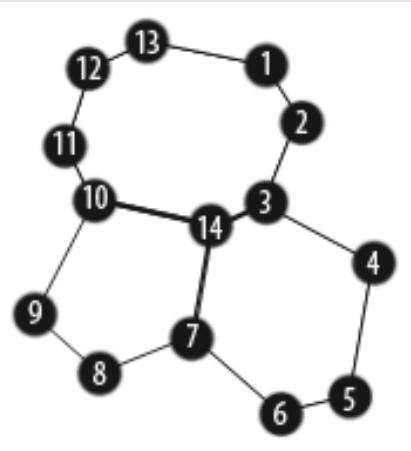

$\mathrm{G}_{676}=\left(\mathrm{V}_{676}, \mathrm{~A}_{676}\right)$

M. pallidipennis

$\mathrm{G}_{865}=\left(\mathrm{V}_{865}, \mathrm{~A}_{865}\right)$

T. infestans
$\mathrm{V}_{676}=\{1, \ldots \ldots ., 14\}$

$\mathrm{A}_{676}=\{\{1,2\},\{2,3\},\{3,4\},\{4,5\},\{5,6\},\{6,7\}$, $\{7,8\},\{8,9\},\{9,10\},\{10,11\},\{11,12\},\{12,13\}$, $\{13,1\},\{2,14\},\{7,14\},\{11,14\}\}$

$\mathrm{A}_{865}=\{\{1,2\},\{2,3\},\{3,4\},\{4,5\},\{5,6\},\{6,7\}$, $\{7,8\},\{8,9\},\{9,10\},\{10,11\},\{11,12\},\{12,13\}$, $\{13,1\},\{3,14\},\{7,14\},\{10,14\}\}$

$\mathrm{V}_{476}=\{1, \ldots \ldots ., 12\}$

$\mathrm{G}_{476}=\left(\mathrm{V}_{476}, \mathrm{~A}_{476}\right)$

T. infestans
$\mathrm{A}_{476}=\{\{1,2\},\{2,3\},\{3,4\},\{4,5\},\{5,6\},\{6,7\}$, $\{7,8\},\{8,9\},\{9,10\},\{10,11\},\{11,1\},\{1,12\}$, $\{6,12\},\{10,12\}\}$

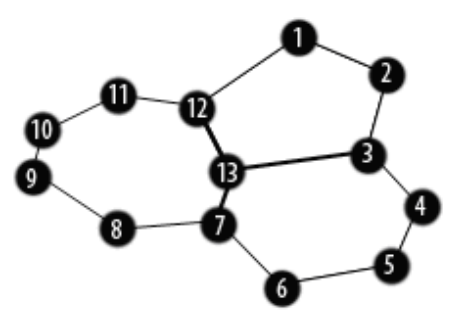

$\mathrm{V}_{567}=\{1, \ldots \ldots ., 13\}$

$\mathrm{G}_{567}=\left(\mathrm{V}_{567}, \mathrm{~A}_{567}\right)$

$\mathrm{A}_{567}=\{\{1,2\},\{2,3\},\{3,4\},\{4,5\},\{5,6\},\{6,7\}$,

T. infestans $\{7,8\},\{8,9\},\{9,10\},\{10,11\},\{11,12\},\{12,1\}$, $\{3,13\},\{7,13\},\{12,13\}\}$ 

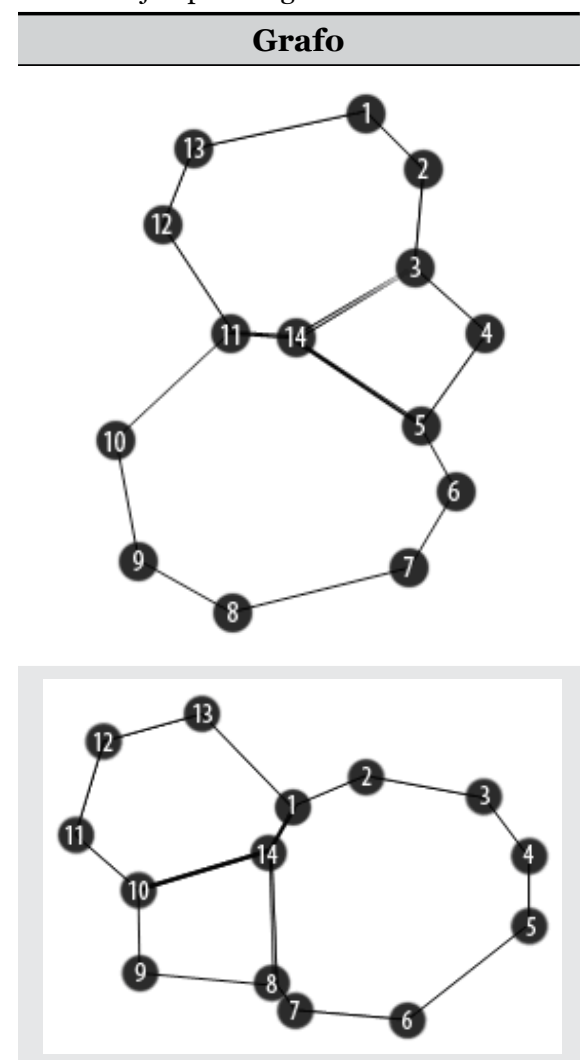

$$
\mathrm{G}_{748}=\left(\mathrm{V}_{748,}, \mathrm{~A}_{748}\right)
$$

M. longipennis

$$
\begin{gathered}
\mathrm{G}_{946}=\left(\mathrm{V}_{946}, \mathrm{~A}_{946)}\right. \\
\text { T. lenti }
\end{gathered}
$$$$
\mathrm{V}_{946}=\{1, \ldots \ldots ., 14\}
$$

A946 $=\{\{1,2\},\{2,3\},\{3,4\},\{4,5\},\{5,6\},\{6,7\}$, $\{7,8\},\{8,9\},\{9,10\},\{10,11\},\{11,12\},\{12,13\}$, $\{13,1\},\{1,14\},\{8,14\},\{10,14\}\}$

$\mathrm{A}_{748}=\{\{1,2\},\{2,3\},\{3,4\},\{4,5\},\{5,6\},\{6,7\}$, $\{7,8\},\{8,9\},\{9,10\},\{10,11\},\{11,12\},\{12,13\}$, $\{13,1\},\{3,14\},\{5,14\},\{11,14\}\}$

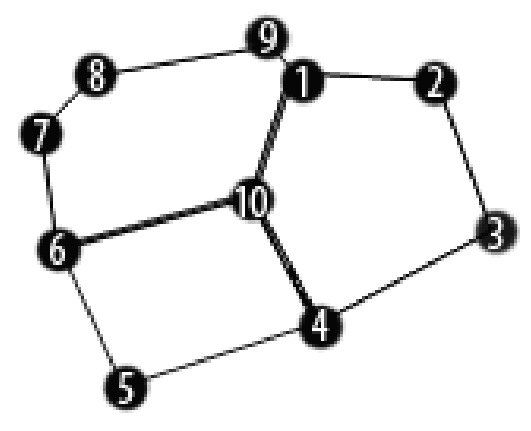

$\mathrm{V}_{748}=\{1, \ldots \ldots ., 14\}$

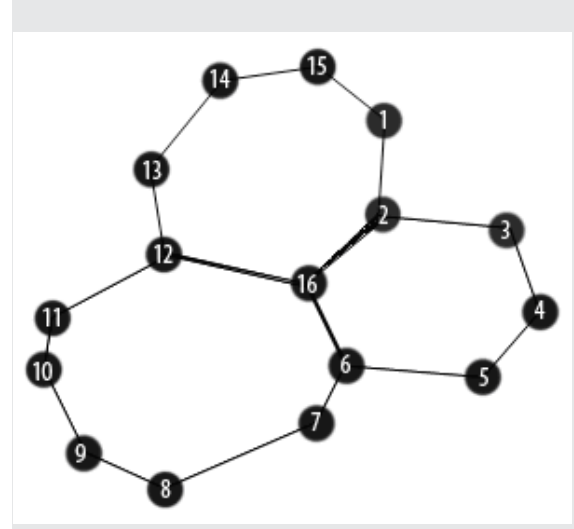

$$
\begin{gathered}
\mathrm{G}_{546}=\left(\mathrm{V}_{546}, \mathrm{~A}_{546}\right) \\
\text { T. maculata }
\end{gathered}
$$

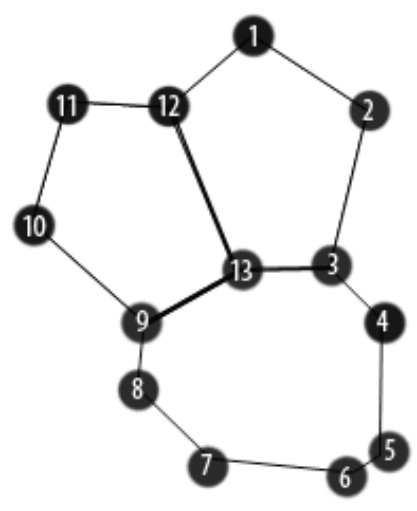

$\mathrm{G}_{575}=\left(\mathrm{V}_{575}, \mathrm{~A}_{575}\right)$

M. pallidipennis
$\mathrm{V}_{546}=\{1, \ldots \ldots ., 10\}$

$\mathrm{A}_{546}=\{\{1,2\},\{2,3\},\{3,4\},\{4,5\},\{5,6\},\{6,7\}$,

$\{7,8\},\{8,9\},\{9,1\},\{1,10\},\{4,10\},\{6,10\}$
$\mathrm{V}_{768}=\{1, \ldots \ldots, 16\}$

$\mathrm{G}_{768}=\left(\mathrm{V}_{768}, \mathrm{~A}_{768}\right)$

T. maculata

$\mathrm{A}_{768}=\{\{1,2\},\{2,3\},\{3,4\},\{4,5\},\{5,6\},\{6,7\}$, $\{7,8\},\{8,9\},\{9,10\},\{10,11\},\{11,12\},\{12,13\}$, $\{13,14\},\{14,15\},\{15,1\},\{2,16\},\{6,16\},\{12,16\}\}$

$\mathrm{V}_{575}=\{1, \ldots \ldots ., 13\}$

$\mathrm{A}_{575}=\{\{1,2\},\{2,3\},\{3,4\},\{4,5\},\{5,6\},\{6,7\}$, $\{7,8\},\{8,9\},\{9,10\},\{10,11\},\{11,12\},\{12,1\}$, $\{3,13\},\{9,13\},\{12,13\}\}$ 
Tabla 7. Ejemplos de grafos observados en las especies T. sordida, M. pallidipennis, T. infestans y M. picturatus

\begin{tabular}{|c|c|c|}
\hline Grafo & Notación & Elementos \\
\hline & $\begin{array}{c}\mathrm{G}_{577}=\left(\mathrm{V}_{577}, \mathrm{~A}_{577}\right) \\
\text { T. sordida }\end{array}$ & $\begin{array}{l}\mathrm{V}_{577}=\{1, \ldots \ldots, 14\} \\
\mathrm{A}_{577}=\{\{1,2\},\{2,3\},\{3,4\},\{4,5\},\{5,6\},\{6,7\}, \\
\{7,8\},\{8,9\},\{9,10\},\{10,11\},\{11,12\},\{12,13\},\{13,1\},\{3,14\},\{8 \\
, 14\},\{13,14\}\}\end{array}$ \\
\hline
\end{tabular}

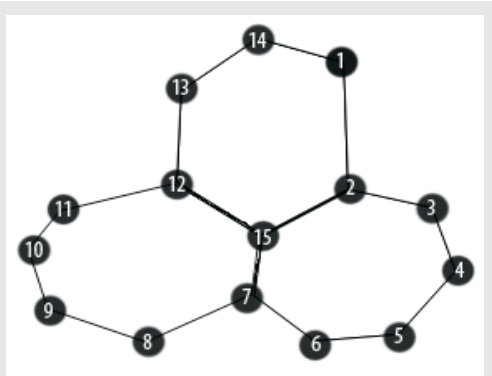

$\mathrm{V}_{677}=\{1, \ldots \ldots, 15\}$

$\mathrm{G}_{677}=\left(\mathrm{V}_{677}, \mathrm{~A}_{677}\right)$

M. pallidipennis

$\mathrm{A}_{677}=\{\{1,2\},\{2,3\},\{3,4\},\{4,5\},\{5,6\},\{6,7\}$,

$\{7,8\},\{8,9\},\{9,10\},\{10,11\},\{11,12\},\{12,13\},\{13,14\},\{14,1\},\{$ $2,15\},\{7,15\},\{12,15\}\}$

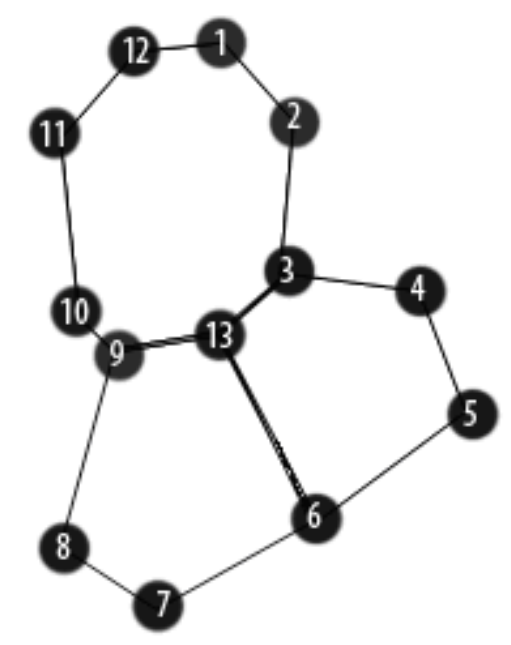

$\mathrm{V}_{855}=\{1, \ldots \ldots ., 13\}$

$\mathrm{G}_{855}=\left(\mathrm{V}_{855}, \mathrm{~A}_{855}\right)$

T. sordida
$\mathrm{A}_{855}=\{\{1,2\},\{2,3\},\{3,4\},\{4,5\},\{5,6\},\{6,7\}$, $\{7,8\},\{8,9\},\{9,10\},\{10,11\},\{11,12\},\{12,1\}$, $\{3,13\},\{6,13\},\{9,13\}\}$

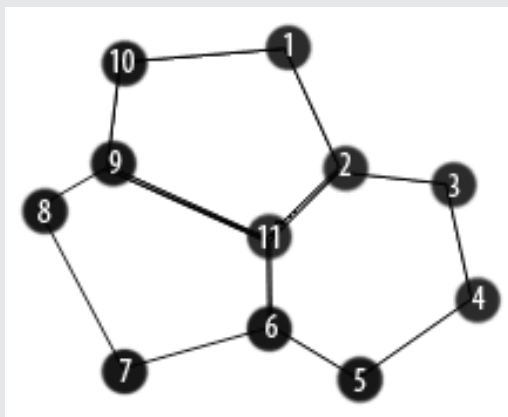

$\mathrm{V}_{565}=\{1, \ldots \ldots, 10\}$

$\mathrm{G}_{565}=\left(\mathrm{V}_{565}, \mathrm{~A}_{565}\right)$

T. infestans
$\mathrm{A}_{565}=\{\{1,2\},\{2,3\},\{3,4\},\{4,5\},\{5,6\},\{6,7\}$, $\{7,8\},\{8,9\},\{9,10\},\{10,1\},\{2,11\},\{6,11\}$, $\{9,11\}\}$

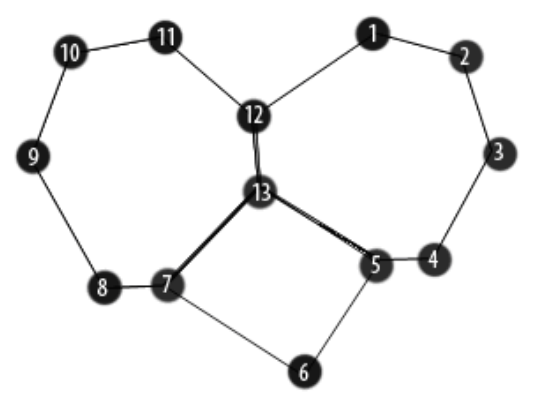

$\mathrm{V}_{747}=\{1, \ldots \ldots ., 13\}$

$\mathrm{G}_{747}=\left(\mathrm{V}_{747}, \mathrm{~A}_{747}\right)$

M. picturatus
$\mathrm{A}_{747}=\{\{1,2\},\{2,3\},\{3,4\},\{4,5\},\{5,6\},\{6,7\}$, $\{7,8\},\{8,9\},\{9,10\},\{10,11\},\{11,12\},\{12,1\}$, $\{5,13\},\{7,13\},\{12,13\}\}$ 


Grafo
Notación

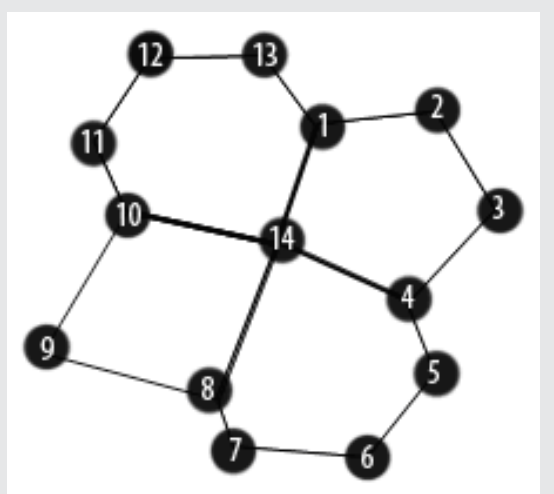

$\mathrm{V}_{5646}=\{1, \ldots \ldots, 14\}$

$$
\begin{gathered}
\mathrm{G}_{5646}=\left(\mathrm{V}_{5646}, \mathrm{~A}_{5646}\right) \\
\text { T. lenti }
\end{gathered}
$$

$\mathrm{A}_{5646}=\{\{1,2\},\{2,3\},\{3,4\},\{4,5\},\{5,6\}$,

$\{6,7\},\{7,8\},\{8,9\},\{9,10\},\{10,11\},\{11,12\}$, $\{12,13\},\{13,1\},\{4,14\},\{8,14\},\{10,14\}$, $\{1,14\}\}$

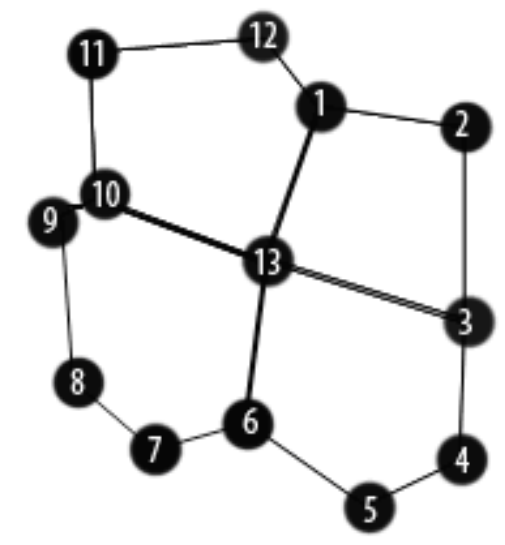

$\mathrm{V}_{6456}=\{1, \ldots \ldots ., 13\}$

$$
\mathrm{G}_{6456}=\left(\mathrm{V}_{6456}, \mathrm{~A}_{6456}\right)
$$

T. sordida
$\mathrm{A}_{6456}=\{\{1,2\},\{2,3\},\{3,4\},\{4,5\},\{5,6\}$, $\{6,7\},\{7,8\},\{8,9\},\{9,10\},\{10,11\},\{11,12\}$, $\{12,1\},\{3,13\},\{6,13\},\{10,13\},\{1,13\}\}$

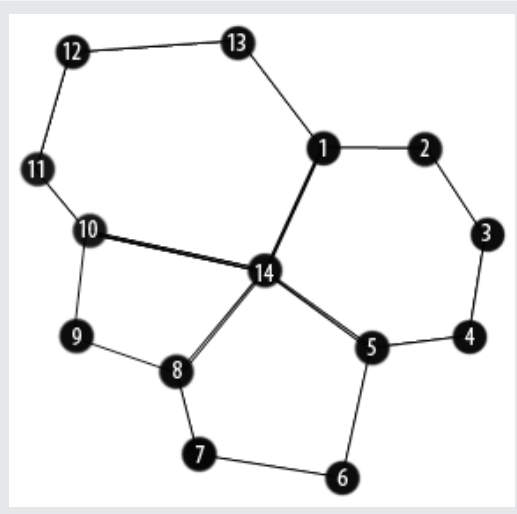

$\mathrm{V}_{6654}=\{1, \ldots \ldots ., 14\}$

$\mathrm{G}_{6654}=\left(\mathrm{V}_{6654}, \mathrm{~A}_{6654}\right)$

M. phyllososmus

$\mathrm{A}_{6654}=\{\{1,2\},\{2,3\},\{3,4\},\{4,5\},\{5,6\}$, $\{6,7\},\{7,8\},\{8,9\},\{9,10\},\{10,11\},\{11,12\}$, $\{12,13\},\{13,1\},\{5,14\},\{8,14\},\{10,14\}$, $\{1,14\}\}$

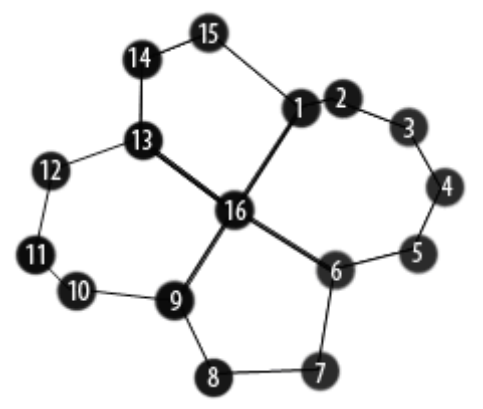

$\mathrm{V}_{5756}=\{1, \ldots \ldots, 16\}$

$\mathrm{G}_{5756}=\left(\mathrm{V}_{5756}, \mathrm{~A}_{5756}\right)$

T. lenti
$\mathrm{A}_{5756}=\{\{1,2\},\{2,3\},\{3,4\},\{4,5\},\{5,6\}$, $\{6,7\},\{7,8\},\{8,9\},\{9,10\},\{10,11\},\{11,12\}$, $\{12,13\},\{13,14\},\{14,15\},\{15,1\},\{6,16\}$, $\{9,16\},\{13,16\},\{1,16\}\}$ 


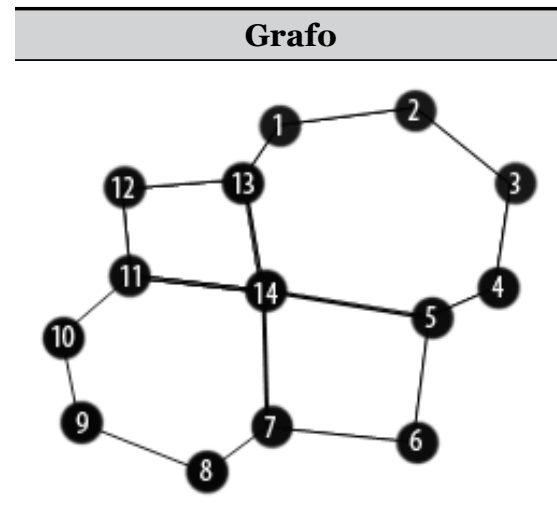

$$
\begin{gathered}
\mathrm{G}_{7464}=\left(\mathrm{V}_{7464}, \mathrm{~A}_{7464}\right) \\
\text { T. lenti }
\end{gathered}
$$

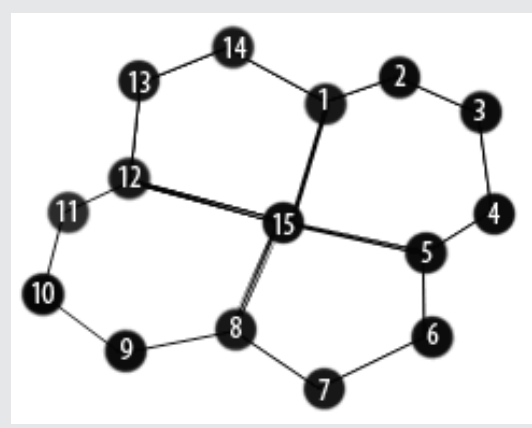

$$
\mathrm{G}_{6565}=\left(\mathrm{V}_{6565}, \mathrm{~A}_{6565}\right)
$$

T. infestans

$$
\mathrm{G}_{5566}=\left(\mathrm{V}_{5566}, \mathrm{~A}_{5566}\right)
$$

M. longipennis
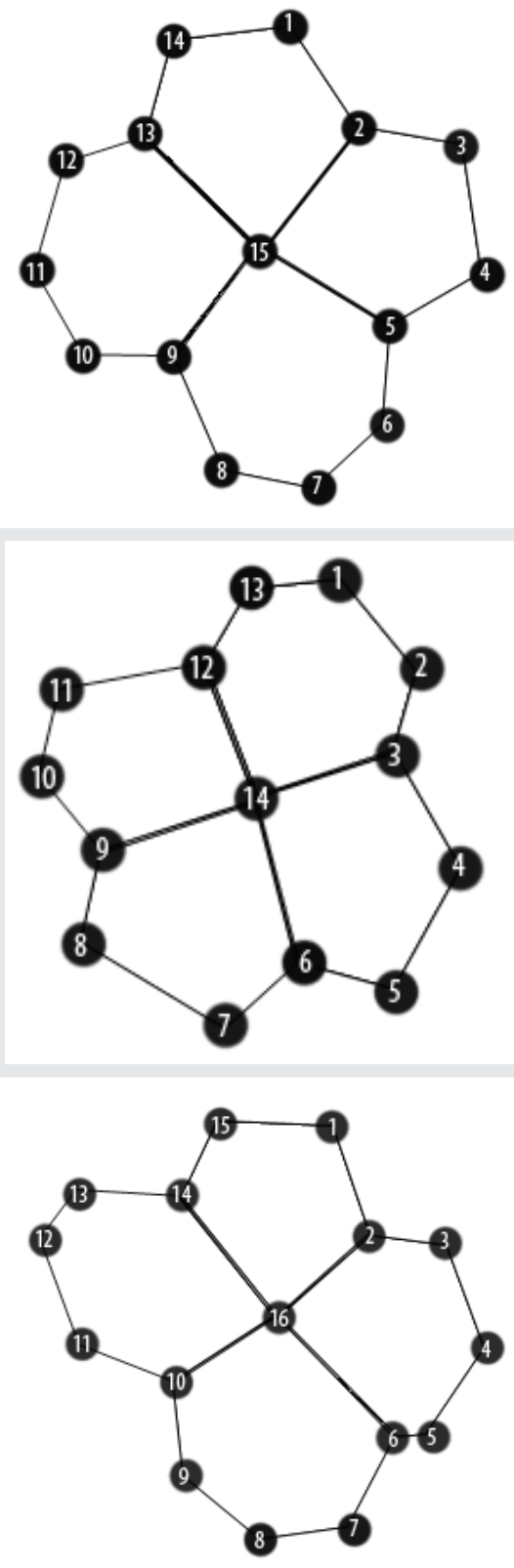

$$
\mathrm{G}_{6555}=\left(\mathrm{V}_{6555}, \mathrm{~A}_{6555}\right)
$$

N. flavida

$$
\mathrm{G}_{5666}=\left(\mathrm{V}_{5666}, \mathrm{~A}_{5666}\right)
$$

T. lenti$$
\mathrm{V}_{7464}=\{1, \ldots \ldots ., 14\}
$$

$\mathrm{A}_{7464}=\{\{1,2\},\{2,3\},\{3,4\},\{4,5\},\{5,6\},\{6,7\}$, $\{7,8\},\{8,9\},\{9,10\},\{10,11\},\{11,12\},\{12,13\}$, $\{13,1\},\{5,14\},\{7,14\},\{11,14\},\{13,14\}\}$

Elementos

$\mathrm{V}_{6565}=\{1, \ldots \ldots ., 15\}$

$\mathrm{A}_{6565}=\{\{1,2\},\{2,3\},\{3,4\},\{4,5\},\{5,6\},\{6,7\}$,

$\{7,8\},\{8,9\},\{9,10\},\{10,11\},\{11,12\},\{12,13\},\{13,14\}$, $\{14,1\},\{5,15\},\{8,15\},\{12,15\},\{1,15\}\}$
$\mathrm{A}_{5566}=\{\{1,2\},\{2,3\},\{3,4\},\{4,5\},\{5,6\},\{6,7\}$,

$\{7,8\},\{8,9\},\{9,10\},\{10,11\},\{11,12\},\{12,13\},\{13,14\}$, $\{14,1\},\{2,15\},\{5,15\},\{9,15\},\{13,15\}\}$
$\mathrm{V}_{6555}=\{1, \ldots \ldots ., 14\}$

$\mathrm{A}_{6555}=\{\{1,2\},\{2,3\},\{3,4\},\{4,5\},\{5,6\},\{6,7\}$, $\{7,8\},\{8,9\},\{9,10\},\{10,11\},\{11,12\},\{12,13\},\{13,1\}$, $\{3,14\},\{6,14\},\{9,14\},\{12,14\}\}$

$$
\mathrm{V}_{5666}=\{1 \ldots \ldots . .16\}
$$

$\mathrm{A}_{5666}=\{\{1,2\},\{2,3\},\{3,4\},\{4,5\},\{5,6\},\{6,7\}$,

$\{7,8\},\{8,9\},\{9,10\},\{10,11\},\{11,12\},\{12,13\},\{13,14\}$, $\{14,15\},\{15,1\},\{2,16\},\{6,16\},\{10,16\},\{14,16\}\}$ 


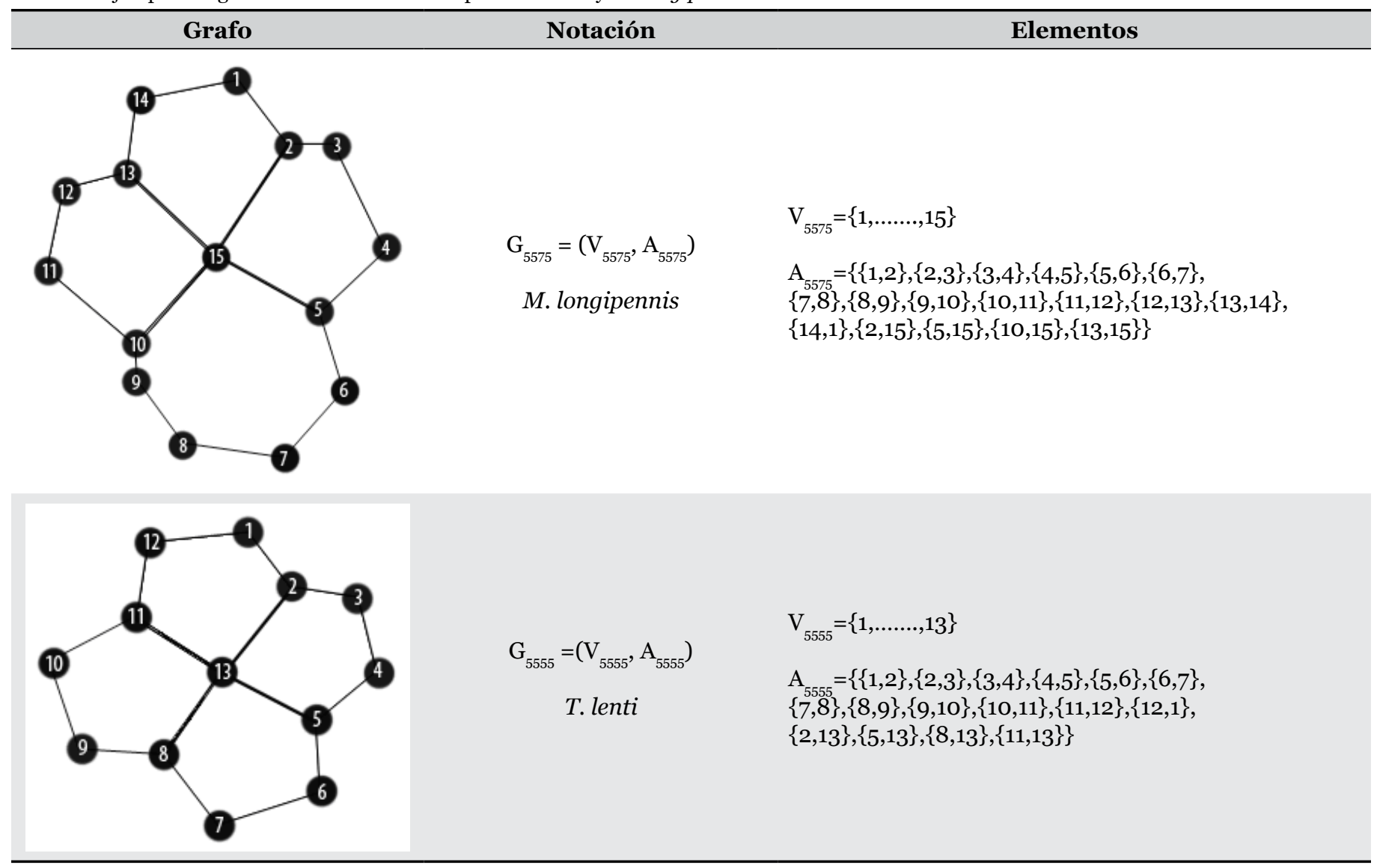

\section{AGRADECIMIENTOS}

Este trabajo fue financiado parcialmente por el proyecto CDCHT-ULA1665-09-03-F.AAndrés Moray Jorge Fernández del LAQUEM, integrantes del Laboratorio Nacional de Microscopía y Microanálisis (Proyecto FONACIT Lab-2001001442), a Arol Rojas y Fernando Otálora. Los resultados del presente trabajo forman parte del Trabajo Especial de Grado del primer autor.

\section{REFERENCIAS}

Barata, J. 1981. Aspectos morfológicos de ovos de Triatominae II. Características macroscópicas e exocoriais de dez espécies do género Rhodnius Stal, 1859 (Hemiptera: Reduviidae). Revista de Saúde pública,15: 490-542.

Barata, J. 1998. Estructuras macroscópicas e exocoriais de ovos de Triatominae (Hemiptera, Reduviidae), p. 409-448. En: Carcavallo R.U., I. Galíndez Girón, J. Jurberg \& H. Lent (Eds). Atlas dos Vectores da Doença de Chagas nas Américas. Rio de Janeiro, Editorial Fiocruz, 1217p.

Barth, R. \& H. Muth, 1958. Estudos anatômicos e histológicos sobre a subfamília Triatominae (Heteroptera, Reduviidae). VIII parte: Observações sobre a superfície dos ovos das espécies mais importantes. Memórias do Instituto Oswaldo Cruz, 56 (1):197-208.

Beament, J. W. L., 1946. The Formation and Structure of the Chorion of the Egg in an Hemipteran, Rhodnius prolixus. Quarterly Journal of Microscopical Science, s2-87(348): 393439.

Briceño-Iragorry, L. 1934.Contribución al estudio morfológico de los huevos de algunos Reduvideos. Boletín de la Sociedad Venezolana de Ciencias Naturales, 2: 397-400.

Carcavallo, R.U., J. Jurberg \& H. Lent. 1998. Filogenia dos Triatomíneos, p 925-969. En: Carcavallo, R.U., I. Galíndez. Girón, J. Jurberg \& H. Lent (Eds). Atlas dos Vectores da Doença de Chagas nas Américas. Rio de Janeiro, Editorial Fiocruz, 1217p.

Castro, N., J. Cobos, J. C. Dana \& A. Márquez, 1998. Teselaciones y Grafos de Intersección de Segmentos en Superficies no Planas. Universidad de Sebilla,
Departamento de matematica aplicada I;1-9. Disponible en: http://ma1.eii.us.es/miembros/dana/articulo/Gerona/ Tgissp.pdf.

Chaves, L.F \& N. Añez, 2003. Geometría de las células del exocorión de huevos de Rhodnius prolixus Stal, 1859 (Heteroptera: Reduviidae). Entomotrópica, 18(1):1-5.

Costa, J., O.M. Barth, V. Marchon-Silva, C.E. De Almeida, R.M. G. Freitas-Sibajev \& F. Panzera, 1997. Morphological Studies on the Triatoma brasiliensis Neiva, 1911 (Hemiptera, Reduviidae, Triatominae) Genital Structures and Eggs of Different Chromatic Forms. Memórias do Instituto Oswaldo Cruz, Rio de Janeiro, 92(4):493-498.

Diestel, R. 2005. Graph Theory, 410p. Disponible en: http://diestel-graph-theory.com/GrTh.html.

Galvão, C., R. Carcavallo, D. Rocha \& J. Jurberg, 2003. A checklist of the current valid species of the subfamily Triatominae Jeannel, 1919 (Hemiptera, Reduviidae) and their geographical distribution, with nomenclatural and taxonomic notes. Zootaza, 202: 1-36.

García, B. 1998. Relações Filogenéticas Moleculares entre Espécies do Gênero Triatoma, p. 971-980. En: Carcavallo, R.U., I.Galíndez. Girón, J. Jurberg \& H. Lent (Eds). Atlas dos Vectores da Doença de Chagas nas Americas. Rio de Janeiro, Editorial Fiocruz, 1217p.

Gonçalves, M. T., J. Jurberg, J.M. Costa \& W. De Souza, 1985. Estudo Morfológico Comparativo de Ovos e Ninfas de Triatoma maculata (Erichson, 1848) e Triatoma pseudomaculata Correa \& Espínola, 1964 (Hemiptera, Reduviidae, Triatominae). Memórias do Instituto Oswaldo Cruz, Rio de Janeiro, 80(3):263-276.

Hinton, H. E \& M. W. Service, 1969. The surface structure of aedine eggs as seen with the scanning electron microscope. Annals of Tropical Medicine and Parasitology, 63(4):409411.

Hypša, V., F. D. Tietz, J. Zrzavý, R. O. M. Rego, C. Galvão \& J. Jurberg, 2002. Phylogeny and biogeography of Triatominae (Hemiptera: Reduviidae): molecular evidence of a new World origin of the Asiatic clade. Molecular Phylogenetics and Evolution, 23:447-457. 
Lent, H. \& P. Wygodzinsky, 1979. Revision of the Triatominae (Hemiptera, Reduviidae), and their significance as vectors of Chagas disease. Bulletin of the American Museum of Natural History, 163(3): 123-520p.

Lucena, D. T. \& A. M. Rego, 1969. Microestrutura do Exocório de ovos de Triatomíneos do Nordeste do Brasil. Revista do Instituto de Medicina Tropical de São Paulo, 11(2):87-93.

Obara, M. T., J. M. S Barata, N. N. da Silva, W. C. Júnior, P. R. Urbinatti, J. A. da Rosa, J. Jurberg \& C. Galvão, 2007a. Estudo de ovos de quatro espécies do gênero Meccus (Hemiptera, Reduviidae, Triatominae), vectores da doença de Chagas. Memórias do Instituto Oswaldo Cruz, Rio de Janeiro, 102(1): 13-19.

Obara, M. T., J. A. Da Rosa, N. N. Da Silva, W. Jr. Ceretti, P. R Urbinatti, J. M. S Barata, J. Jurberg \& C. Galvão, 2007b. Estudo Morfológico e Histológicos dos Ovos de Seis Espécies do Gênero Triatoma (Hemiptera, Reduviidae). Neotropical Entomology, 36(5):798-806.

Rozen, J.G. \& H. Özbek, 2005. Notes on the Egg and Egg Deposition of the Cleptoparasite Thyreus ramosus (Hymenoptera: Apidae: Melectini). Journal of the Kansas Entomological Society, 78(1):34-40.

Selivon, D. \& A. L. P. Perondini, 1999. Description of Anastrepha sororcula and $A$. serpentina (Diptera: Tephritidae) eggs. Florida Entomologist, 82(2):347-353.

\section{Como citar este artigo:}

González, G., E. Aldana, E. Lizano \& G. López, 2009. Arreglo de los polígonos del exocorion de huevos eclosionados de algunas especies de los géneros Triatoma Laporte, Meccus Stal y Nesotriatoma Usinger (Heteroptera: Reduviidae). EntomoBrasilis, 2(3): 76-89. www.periodico.ebras.bio.br/ojs
Silva, A. M., J. Jurberg, H.S. Barbosa, D.S. Rocha, R.U. Carcavallo \& C. Galvão, 2005. Morfologia comparada dos ovos e ninfas de Triatoma vandae Carcavallo, Jurberg, Rocha, Galvão, Noireau \& Lent, 2002 e Triatoma williami Galvão, Souza \& Lima, 1965 (Hemiptera, Reduviidae, Triatominae). Memórias do Instituto Oswaldo Cruz, Rio de Janeiro, 100(6):649-661.

Tamassia , R. \& I. G. Tollis, 1986. Tesselletion representations of planar graphs. Proc. 27th Annual Allerton Conference, p.4857.

Tartarotti, E., V. T. M. Azeredo-Oliveira \& R. C Ceron, 2006. Phylogenetic approach to the study of Triatomines (Triatominae, Heteroptera). Brazilian Journal of Biology, 66(2B):703-708.

Visciarelli, E., A. Ferrero \& S. R. Costamagna, 2004. Aspectos Exocoriales de Huevos de Triatoma patagonica Del Ponte, 1929 por Microscopía Electrónica de Barrido. Entomología y Vectores, 11(4):653-668.

Wigglesworth, V. 1972. The Principes of Insect Physiology. Seventh Edition, Halsted Press UK: p.827.

Recebido em: 12/06/2009

Aceito em: 30/11/2009

$* * * * * * * * * * * * *$
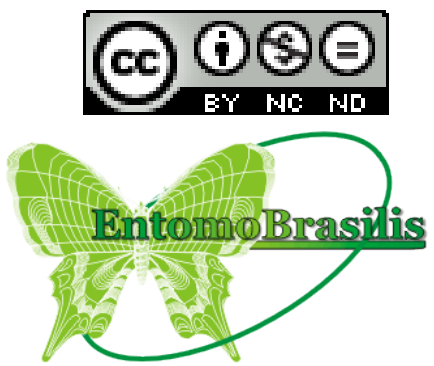